\title{
Spectroscopic classification and Gaia DR2 parallaxes of new nearby white dwarfs among selected blue proper motion stars ${ }^{\star}$
}

\author{
R.-D. Scholz ${ }^{1}$, H. Meusinger ${ }^{2,3}$, A. Schwope ${ }^{1}$, H. Jahrei $\beta^{4}$, and I. Pelisoli ${ }^{5,6}$ \\ ${ }^{1}$ Leibniz-Institut für Astrophysik Potsdam, An der Sternwarte 16, 14482 Potsdam, Germany \\ e-mail: rdscholz@aip.de \\ 2 Thüringer Landessternwarte Tautenburg, Sternwarte 5, 07778 Tautenburg, Germany \\ 3 University Leipzig, Faculty of Physics and Geosciences, Linnèstr. 5, 04103 Leipzig, Germany \\ 4 Zentrum für Astronomie der Universität Heidelberg, Astronomisches Rechen-Institut, Mönchhofstraße 12-14, \\ 69120 Heidelberg, Germany \\ 5 Instituto de Física, Universidade Federal do Rio Grande do Sul, 91501-900 Porto-Alegre, RS, Brazil \\ ${ }^{6}$ Institut für Physik und Astronomie, Universitätsstandort Golm, Haus 28, Karl-Liebknecht-Str. 24/25, 14467 Potsdam, Germany
}

Received 22 June 2018 / Accepted 6 August 2018

\begin{abstract}
Aims. With our low-resolution spectroscopic observing programme for selected blue proper motion stars, we tried to find new white dwarfs (WDs) in the solar neighbourhood.

Methods. We used the Lépine \& Shara Proper Motion (LSPM) catalogue with a lower proper motion limit of $150 \mathrm{mas} \mathrm{yr}^{-1}$ and $^{-1}$ the Second US Naval Observatory CCD Astrograph Catalog (UCAC2) for proper motions down to about $90 \mathrm{mas} \mathrm{yr}^{-1}$. The LSPM and UCAC2 photometry was combined with Two Micron All Sky Survey (2MASS) near-infrared (NIR) photometry. Targets selected according to their blue optical-to-NIR and NIR colours were observed mainly at Calar Alto. The spectra were classified by comparison with a large number of already known comparison objects, including WDs, simultaneously observed within our programme. Gaia DR2 parallaxes and colours were used to confirm or reject spectroscopic WD candidates and to derive improved effective temperatures. Results. We found ten new WDs at distances between $24.4 \mathrm{pc}$ and $79.8 \mathrm{pc}$, including six hot DA WDs: GD 221 (DA2.0), HD 166435 B (DA2.2), GD 277 (DA2.2), 2MASS J19293865+1117523 (DA2.4), 2MASS J05280449+4105253 (DA3.6), and 2MASS J050051850930549 (DA4.2). The latter is rather bright $(G \approx 12.6)$ and with its Gaia DR2 parallax of $\approx 14$ mas it appears overluminous by about 3 mag compared to the WD sequence in the Gaia DR2 colour-magnitude diagram. It may be the closest extremely low mass (ELM) WD to the Sun. We further classified 2MASS J07035743+2534184 as DB4.1. With its distance of $25.6 \mathrm{pc}$ it is the second nearest known representative of its class. With GD 28 (DA6.1), LP 740-47 (DA7.5), and LSPM J1919+4527 (DC10.3) three additional cool WDs were found. Gaia DR2 parallaxes show that four of our candidates, but also two previously supposed WDs (WD 1004+665 and LSPM J1445+2527) are in fact distant Galactic halo stars with high tangential velocities. Among our rejected WD candidates, we identified a bright ( $G=13.4 \mathrm{mag}$ ) G-type carbon dwarf, LSPM J0937+2803, at a distance of $272 \mathrm{pc}$.
\end{abstract}

Key words. techniques: spectroscopic - surveys - parallaxes - proper motions - white dwarfs - solar neighborhood

\section{Introduction}

The stellar content of the solar neighbourhood provides important information for the study of the stellar initial mass function, Galactic structure and evolution. White dwarfs (WDs) play an important role in our understanding of these fundamental astrophysical issues (Reid 2005; Tremblay et al. 2014). In particular, the cool WDs provide age estimates of the Galactic disk (Gianninas et al. 2015; Kilic et al. 2017) and information on the baryonic dark matter in the Galaxy (Reid et al. 2001; Méndez 2002; Flynn et al. 2003). The nearest WDs are also excellent targets for divers methods to search for extrasolar planets (Kilic et al. 2010; Xu et al. 2015).

As the general census of nearby stars has been slowly improved in the pre-Gaia era (see e.g. Henry et al. 2018) the sample of known WDs in the solar neighbourhood experienced a similar evolution. While the WD census was believed to be complete within $13 \mathrm{pc}$ (Holberg et al. 2016), during the last ten years many efforts were undertaken to identify more

\footnotetext{
* Based on observations with the $2.2 \mathrm{~m}$ telescope of the Centro Astronómico Hispano-Aleman (CAHA), Calar Alto, Spain.
}

WDs in the extended solar neighbourhood (for instance for the $25 \mathrm{pc}$ and $40 \mathrm{pc}$ samples) by measuring their parallaxes (Subasavage et al. 2009, 2017) and using spectroscopic surveys (Gianninas et al. 2011; Kawka \& Vennes 2012; Limoges et al. 2013; Sion et al. 2014). The physical properties of nearby WDs were investigated in more detail by Giammichele et al. (2012), Limoges et al. (2015), Tremblay et al. (2017), Hollands et al. (2017), and Skinner et al. (2017). The overall number of spectroscopically classified WDs (including more distant objects) was increased fivefold with the catalogues of Kleinman et al. (2013) and Kepler et al. (2015, 2016), based on spectroscopic data from the Sloan Digital Sky Survey (SDSS; York et al. 2000).

Despite of all the above mentioned efforts, even the generally assumed completeness of the $13 \mathrm{pc}$ WD sample was shown to be not correct, as the recent discovery of the cool WD companion of a new nearby red dwarf star TYC 3980-1081-1 B (Scholz et al. 2018) at a distance of $8.3 \mathrm{pc}$ indicated. This discovery was based on astrometric data from Gaia DR1 (Lindegren et al. 2016), the 5th United States Naval Observatory CCD Astrograph Catalog (UCAC5; Zacharias et al. 2017), and the U.S. Naval Observatory Robotic Astrometric Telescope (URAT; Zacharias et al. 2015) 
Parallax Catalog (UPC; Finch \& Zacharias 2016b). The WD status of TYC 3980-1081-1 B is now confirmed by its Gaia DR2 (Gaia Collaboration 2018b) parallax of $118.12 \pm 0.02$ mas.

Most searches for previously unrecognised nearby stars in the pre-Gaia era investigated targets selected from combined colour- and proper motion surveys. In 2008, we started a spectroscopic follow-up programme to search for missing WDs in the solar neighbourhood in a sample of blue proper motion stars. The Lépine \& Shara Proper Motion (LSPM; Lépine \& Shara 2005) catalogue of the northern sky and the Second US Naval Observatory CCD Astrograph Catalog (UCAC2; Zacharias et al. 2004) covering the sky area from $-90^{\circ}$ to $+40^{\circ}$ declination (going up to $+52^{\circ}$ in some areas), and the Two Micron All Sky Survey (2MASS; Skrutskie et al. 2006) providing accurate near-infrared (NIR) photometry served as our main input data with respect to proper motions and photometry. The results of our WD survey were not published so far, except for one new WD (HD 166435 $\mathrm{B}=\mathrm{CA} 376)$ that was independently discovered in a search for common proper motion WD companions of known nearby stars (Scholz et al. 2018).

This paper is organised as follows: in Sect. 2 we describe the selection of our target stars for the spectroscopic observations, which were mainly carried out at the Calar Alto observatory (Sect. 3). Section 4 deals with our spectroscopic classification of WD candidates of different classes, whereas Sects. 5 and 6 show how well our results are confirmed by Gaia DR2 parallaxes and colours. Finally, we review previous investigations on our confirmed and rejected WDs and briefly discuss the properties of the most interesting objects in Sect. 7.

\section{Sample of blue proper motion stars}

For the nearest stars in the $10 \mathrm{pc}$ sample, the Research Consortium on Nearby Stars (RECONS) had reported statistics for the time from 2000 to $2012^{1}$. According to this, the number of WDs increased from 18 to 20, whereas that of other stars (and brown dwarfs) rose from 273 to 337 . It has long been known that in general the nearest known WDs have extraordinary high proper motions compared to the other nearest stars. Using SIMBAD, Scholz et al. (2015) found $84 \%$ of the WDs within $10 \mathrm{pc}$ have proper motions larger than $1000 \mathrm{mas} \mathrm{yr}^{-1}$, whereas the fraction for the other stars was only $49 \%$. Motivated by the discrepancy between the numbers of expected and known WDs, we launched a project to find new WD candidates among stars with moderately high proper motions as surveyed in the LSPM catalogue,

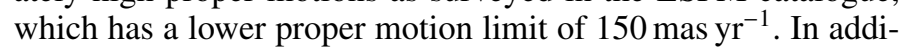
tion, we also selected candidates from the UCAC2 catalogue, which was not the result of a dedicated high proper motion survey but contained relatively accurate proper motions of all stars to about the sixteenth magnitude in a large portion of the sky, to push the proper motion limit for our candidates to even lower values around 100 mas $\mathrm{yr}^{-1}$.

We investigated the distribution of all known WDs within $15 \mathrm{pc}$ compared to all LSPM stars in an optical-to-NIR colourmagnitude diagram (CMD) of $J$ vs. $R F-K_{\mathrm{s}}$, with the NIR magnitudes $J$ and $K_{\mathrm{s}}$ from 2MASS and the photographic red magnitude $R F$ given in the LSPM. We also looked at the pure NIR CMD of $J$ vs. $J-K_{\mathrm{s}}$, providing more accurate colours than the photographically determined LSPM ones. Our colour and magnitude cuts for the selection of new WD candidates corresponded to the observed areas occupied by the known nearby WDs in both of these two CMDs. We decided not to use a reduced

\footnotetext{
1 http://WwW.recons.org/
}

proper motion diagram for the selection of targets, since we were especially interested to find new WDs among the objects with only moderately large proper motions (and small tangential velocities). So our LSPM WD candidates were selected by the following criteria:

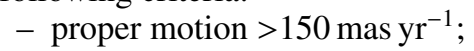

$-K_{\mathrm{s}}<15 \mathrm{mag}$

$-R F-K_{\mathrm{S}}<+1.75 \mathrm{mag}$ and $J-K_{\mathrm{s}}<+0.5 \mathrm{mag}$ if $J>12.5 \mathrm{mag}$;

$-R F-K_{\mathrm{s}}<+0.75 \mathrm{mag}$ and $J-K_{\mathrm{s}}<+0.3 \mathrm{mag}$ if $J<12.5 \mathrm{mag}$. At the beginning of our survey, in 2008, we found that according to SIMBAD bibliography one third of our approximately 600 initial LSPM candidates were already known either as WDs (McCook \& Sion 1999, with updates until 2008) or as Galactic thick disk and halo stars (including uncertain candidates). In particular, all targets with proper motions larger than 500 mas yr $^{-1}$ turned out to be known WDs. Meanwhile these numbers have increased considerably, because new WDs were added mainly thanks to the efforts of Limoges et al. (2013, 2015). They exploited not only the LSPM catalogue but also its unpublished extension to a lower proper motion limit of 40 mas yr$^{-1}$ and used a combination of colour-magnitude and reduced proper motion diagrams in their selection of WD candidates for spectroscopic follow-up observations. Interestingly, 19 out of 54 known WD comparison objects used in our classification (Sect. 4) were spectroscopically classified as WDs only after 2009 , that is after our spectroscopic observing runs.

We selected additional targets with smaller proper motions from the UCAC2 catalogue if they fulfilled the following criteria:

- proper motion $>50$ mas $\mathrm{yr}^{-1}$

- UCmag > $10 \mathrm{mag}$;

- UCmag $-K_{\mathrm{s}}<+1.1 \mathrm{mag}$ and $J-K_{\mathrm{s}}<0.5 \mathrm{mag}$;

- proper motion confirmed with multi-epoch finder charts.

The UCAC2 magnitudes UCmag, with a bandpass between the $V$ and $R$ bands, are probably of higher quality than photographic magnitudes but according to the catalogue description were often observed under non-photometric conditions, and their absolute and relative errors are given to $0.3 \mathrm{mag}$ and $0.1 \mathrm{mag}$. As the UCAC2 contains spurious high proper motion objects, we checked them all by inspecting their multi-epoch positions using the finder chart tool ${ }^{2}$. By this procedure, the number of UCAC2 candidates could be significantly reduced to about 450 objects (including known WDs as well as other known stars according to SIMBAD), especially in crowded regions of the Galactic plane. Compared to the LSPM WD candidates, the UCAC2 WD candidates were given lower priorities the smaller their proper motion is. Effectively, they were only observed if their proper motions were larger than about 90 mas $^{-1} r^{-1}$ and when no other suitable LSPM candidates were available.

\section{Low-resolution spectroscopic observations}

The majority of the known WDs typically show relatively blue colours. Cool WDs, on the other hand, have only moderately blue or even red optical colours (Hansen 1999), which we took into account with our colour cuts described in Sect. 2. By doing so, we expected a substantial contamination of our target sample by high-velocity $F-K$ subdwarfs located at larger distances from the Sun. The adequate method to distinguish WDs from contaminating subdwarfs in a large sample of candidates is low-resolution spectroscopy. This remains true even though

\footnotetext{
2 http://irsa.ipac.caltech.edu/applications/ FinderChart/
} 
observations with low signal-to-noise (S/N) and reaching not far enough to the blue part of the spectrum, where typical atomic (for example lines of $\mathrm{Na}$ and $\mathrm{Ca}$ ) and molecular (for instance the $\mathrm{MgH}$ band at $5200 \AA$ ) absorption features of subdwarfs appear, can be misleading in the classification of cool DC WDs (Sect. 4.4), expected to show featureless spectra, and subdwarfs (compare Scholz et al. 2004; Farihi et al. 2005).

Considering low-resolution stellar spectra dominated by strong Balmer lines, it may also become difficult to distinguish relatively hot DA WDs from so-called sdA stars (Pelisoli et al. $2018 b$ ). The latter are thought to represent various byproducts of binary evolution and include the class of relatively rare extremely low mass (ELM) WDs (Brown et al. 2016). As there are now Gaia parallaxes available, we can estimate absolute magnitudes of such ELM candidates and compare them with those of normal WDs (see Sect. 5).

For the vast majority $(\approx 90 \%)$ of our targets, low-resolution spectra were obtained with the Calar Alto focal reducer and faint object spectrograph (CAFOS) at the $2.2 \mathrm{~m}$ telescope of the Centro Astronómico Hispano-Aleman (CAHA), Calar Alto, Spain. The blue grism B200 was used in combination with the Site-1d CCD resulting in a pixel size corresponding to 0.53 arcsec and a dispersion of $4.7 \AA$ per pixel, or about $15 \AA$ FWHM for a slit width equal to the typical seeing of 1-2 arcsec. For a small subsample of our targets we used the Nasmyth Focal Reducer Spetrograph (NASPEC) at the $2 \mathrm{~m}$ Tautenburg telescope equipped with a Site-T4a CCD chip with a pixel size corresponding to 0.53 arcsec. The V-200 grism was used, which yields a wavelength coverage from $4000 \AA$ to $8500 \AA$ and a dispersion of $3.4 \AA$ per pixel, or $12 \AA$ FWHM for a typical slit width of 1 arcsec.

The results of similar spectroscopic observations with NASPEC and CAFOS of three previously suspected WDs were presented by Jahreiß et al. (2008). All three stars turned out to be high-velocity F- to K-type stars. Interestingly, the low $\mathrm{S} / \mathrm{N}$ NASPEC spectrum of one of these objects (GJ 2091) first pointed at a possible cool WD nature, whereas the CAFOS spectrum (Fig. 2 in Jahreiß et al. 2008) allowed its classification as a distant halo star with a very large tangential velocity $>500 \mathrm{~km} \mathrm{~s}^{-1}$ (now confirmed with Gaia DR2 as $603 \mathrm{~km} \mathrm{~s}^{-1}$ ) and a measured radial velocity of about $270 \mathrm{~km} \mathrm{~s}^{-1}$. In comparison to NASPEC the CAFOS spectroscopy had not only the advantage of usually better observing conditions but also of its extension to the blue spectral range below $4000 \AA$ including the $\mathrm{Ca} \mathrm{H}$ and $\mathrm{K}$ lines, critical for the classification of non-WDs (see Sect. 4.7).

The CAFOS observations were carried out in visitor mode (five nights in August 2008 and five nights in April 2009) by HM and RDS and in service mode (four nights in December 2008, one night in April 2009). The NASPEC observations were made by HM (five nights in February 2008). Exposure times between two and $20 \mathrm{~min}$ (with multiple observations for some of our faintest targets) were sufficient to reach a S/N of at least 10-20 for our targets in the typical magnitude range $12<R F<16$ mag.

The data reduction was performed under ESO MIDAS and followed the standard procedure for long-slit spectroscopy. We used two different software packages, developed for CAFOS and NASPEC spectra. The main differences are in the extraction of one-dimensional spectra and in the flux and wavelength calibration. CAFOS shows substantial distortions of the plate scale near the edges of the field, which is not the case for NASPEC. The CAFOS reduction uses the optimum extraction algorithm by Horne (1986), whereas a simple extraction method was implemented in the NASPEC version. According to our experience, the results from both methods show a good agreement for most stellar spectra (Scholz et al. 2005). The Horne algorithm provides a better $\mathrm{S} / \mathrm{N}$ for the faintest stars, but is more sensitive to variations of the background over the field. Wavelength calibration was done with calibration lamp spectra in the CAFOS version and with the night-sky lines (Osterbrock \& Martel 1992) present in the target spectra for the NASPEC observations. In both versions, a relative flux calibration is implemented that corrects mainly for the spectral sensitivity of the camera. More precise flux calibration was obtained by means of simultaneously observed spectrophotometric standard stars afterwards.

All of the WDs and WD candidates described in this paper were observed with CAFOS. On the other hand, there was no new WD candidate found among the NASPEC spectra. In Sect. 4, we show therefore only CAFOS spectra, which were reduced with the CAFOS reduction package.

As by far not all our candidates could be observed because of bad weather conditions and visibility problems during our scheduled observing runs, our final spectroscopic data set consists of 490 spectra for 410 objects. Multiple observations were carried out for some of the known comparison objects, for some objects that we selected for observations at both Calar Alto and Tautenburg, and for our faintest targets. The 490 spectra finally obtained were sorted by their target coordinates and observing times. All objects were named by their first occurence in that list, with names between "CA001" and "CA490". These designations are used throughout this paper. Additional designations from SIMBAD, in particular of previously known WDs, are given in the corresponding figure captions and in the text. In Tables 1 and 2, we provide also alternative designations for all new and rejected WDs.

\section{WD classification}

Our spectroscopic classification of new WD candidates was mainly based on the direct comparison with known WDs, which were observed with the same instrument. We applied the WD spectral classification system of Sion et al. (1983) illustrated with an atlas of optical WD spectra (Wesemael et al. 1993). All our spectra were normalised to their flux in the wavelength interval 5400-5600 $\AA$ and visually compared with each other, including many different classes of known non-WD objects ( $\mathrm{sdO}, \mathrm{sdB}$, AFG-type stars, low-metallicity F and G subdwarfs). The newly classified non-WD objects will be published in a separate paper (Scholz et al., in prep.), the various new WD types found are described in the following subsections. In most cases our spectra are of sufficient quality for the classification. However, some spectra had only a low $\mathrm{S} / \mathrm{N}$ or no clear matching with the spectra of known comparison objects so that we considered those objects only as WD candidates, to which we assigned spectral types with question marks. The results of our initial spectroscopic classification described in this section are shown in Tables 1 and 2, where we also list our final and more accurate spectral types determined with the aid of Gaia DR2 colours (see Sect. 6).

\subsection{New hot DA WDs and candidates}

Typical hot WDs in the solar neighbourhood, with hydrogen atmospheres and effective temperatures between $10000 \mathrm{~K}$ and $25000 \mathrm{~K}$ (spectral types from $\approx \mathrm{DA} 5.0$ to $\approx \mathrm{DA} 2.0$ ) generally show relatively broad Balmer lines and a blue continuum in their spectra. Compared to A-type stars, the hot DA WDs exhibit a less prominent decline at the Balmer jump, as their higher-level Balmer lines become weaker due to the blanketing effect (Zhang et al. 2013). However, as also mentioned by Zhang et al. (2013), who studied DA WDs in the Large Sky Area Multi-Object Fiber Spectroscopic Telescope (LAMOST) survey 
A\&A 619, A31 (2018)

Table 1. Gaia DR2 astrometry, photometry, and tangential velocities, initial spectral types from CAFOS spectroscopy, and estimated effective temperatures and our corresponding adopted spectral types of ten new WDs from Gaia colours.

\begin{tabular}{|c|c|c|c|c|c|c|c|c|c|c|c|c|}
\hline $\begin{array}{l}\text { CA } \\
\text { No. }\end{array}$ & $\begin{array}{l}\text { Alternative } \\
\text { Names }\end{array}$ & $\begin{array}{r}\mathrm{RA}^{a} \\
\text { (degrees) }\end{array}$ & $\begin{array}{r}\operatorname{Dec}^{a} \\
\text { (degrees) }\end{array}$ & $\begin{array}{l}P l x^{a} \\
\text { (mas) }\end{array}$ & $\begin{array}{r}p m \mathrm{RA}^{a} \\
\left(\mathrm{mas} \mathrm{yr}^{-1}\right)\end{array}$ & $\begin{array}{c}p m \mathrm{Dec}^{a} \\
\left(\mathrm{mas} \mathrm{yr}^{-1}\right)\end{array}$ & $\begin{array}{c}G^{a} \\
(\mathrm{mag})\end{array}$ & $\begin{array}{r}B P-R P^{a} \\
\quad(\mathrm{mag})\end{array}$ & $\begin{array}{c}\text { Initial } \\
\text { SpT }\end{array}$ & $\begin{array}{l}T_{\text {eff }} \\
(\mathrm{K})\end{array}$ & $\begin{array}{l}\text { Adopted } \\
\text { SpT }\end{array}$ & $\begin{array}{r}v_{\tan }^{b} \\
\left(\mathrm{~km} \mathrm{~s}^{-1}\right)\end{array}$ \\
\hline 037 & GD 277 & 22.350203 & +51.145903 & $26.19 \pm 0.05$ & $+32.96 \pm 0.09$ & $-110.58 \pm 0.09$ & 13.550 & -0.338 & $\mathrm{DA} 2.5 \pm 1.0$ & $22857 \pm 2200$ & $\mathrm{DA} 2.2 \pm 0.2$ & 21 \\
\hline 054 & GD 28 & 37.170803 & +36.760490 & $24.41 \pm 0.06$ & $+10.41 \pm 0.10$ & $-171.67 \pm 0.08$ & 15.965 & +0.292 & DA5 $5.0 \pm 2.0 ?$ & $8301 \pm 650$ & DA6. $1 \pm 0.5$ & 33 \\
\hline 078 & 2MASS J05005185-0930549 & 75.215835 & -9.515829 & $13.97 \pm 0.05$ & $-48.55 \pm 0.07$ & $-121.87 \pm 0.07$ & 12.616 & -0.023 & DA $3.0 \pm 1.5 ?$ & $11884 \pm 1100$ & DA $4.2 \pm 0.5$ & 45 \\
\hline 082 & 2MASS J05280449+4105253 & 82.019148 & +41.090348 & $18.31 \pm 0.08$ & $+66.30 \pm 0.10$ & $-3.13 \pm 0.08$ & 15.124 & -0.117 & DA $3.0 \pm 1.0$ & $13994 \pm 1100$ & DA $3.6 \pm 0.3$ & 17 \\
\hline 103 & 2MASS J07035743+2534184 & 105.988994 & +25.571520 & $39.06 \pm 0.03$ & $-58.29 \pm 0.06$ & $-55.69 \pm 0.05$ & 13.912 & -0.037 & DB $3.5 \pm 1.0 ?$ & $12168 \pm 1100$ & DB4. $1 \pm 0.4$ & 10 \\
\hline 305 & LP 740-47 & 220.824701 & -14.621474 & $34.89 \pm 0.08$ & $-225.37 \pm 0.14$ & $-128.43 \pm 0.12$ & 16.218 & +0.585 & DA8.0 \pm 1.0 & $6704 \pm 650$ & $\mathrm{DA} 7.5 \pm 0.8$ & 35 \\
\hline 376 & HD 166435 B & 272.331404 & +29.956111 & $40.90 \pm 0.04$ & $+66.93 \pm 0.06$ & $+74.25 \pm 0.07$ & 13.143 & -0.344 & $\mathrm{DA} 2.5 \pm 1.0$ & $23180 \pm 2200$ & $\mathrm{DA} 2.2 \pm 0.2$ & 12 \\
\hline 397 & LSPM J1919+4527 & 289.900277 & +45.463443 & $35.59 \pm 0.04$ & $-108.92 \pm 0.08$ & $+312.59 \pm 0.09$ & 16.452 & +1.194 & DC10.5 \pm 1.0 & $4901 \pm 300$ & $\mathrm{DC} 10.3 \pm 0.7$ & 44 \\
\hline 399 & GD 221 & 291.858997 & +10.118643 & $12.53 \pm 0.05$ & $-80.04 \pm 0.07$ & $-188.03 \pm 0.06$ & 14.724 & -0.386 & $\mathrm{DA} 2.5 \pm 1.0$ & $25771 \pm 2200$ & $\mathrm{DA} 2.0 \pm 0.2$ & 77 \\
\hline 400 & 2MASS J19293865+1117523 & 292.411127 & +11.298312 & $27.34 \pm 0.05$ & $+19.15 \pm 0.07$ & $+97.83 \pm 0.06$ & 13.508 & -0.313 & $\mathrm{DA} 2.5 \pm 1.0$ & $21445 \pm 2200$ & $\mathrm{DA} 2.4 \pm 0.3$ & 17 \\
\hline
\end{tabular}

Notes. Gaia DR2 coordinates are for (J2000, epoch 2015.5) and were rounded to 0.000001 degrees, parallaxes and their errors were rounded to

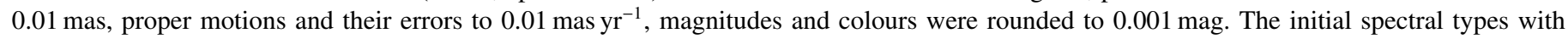
question marks were uncertain because of low $\mathrm{S} / \mathrm{N}$ or possible problems in the flux calibration of the CAFOS spectra. Further notes on the data: ${ }^{(a)}$ Gaia DR2, ${ }^{(b)}$ derived from Gaia DR2 parallaxes and proper motions.

Table 2. Gaia DR2 astrometry, photometry, and tangential velocities, initial (from CAFOS) and adopted spectral types of six rejected WDs.

\begin{tabular}{|c|c|c|c|c|c|c|c|c|c|c|c|c|}
\hline $\begin{array}{l}\text { CA } \\
\text { No. }\end{array}$ & $\begin{array}{l}\text { Alternative } \\
\text { Names }\end{array}$ & $\begin{array}{r}\mathrm{RA}^{a} \\
\text { (degrees) } \\
\end{array}$ & $\begin{array}{r}\operatorname{Dec}^{a} \\
\text { (degrees) } \\
\end{array}$ & $\begin{array}{l}P l x^{a} \\
\text { (mas) }\end{array}$ & $\begin{array}{r}p m \mathrm{RA}^{a} \\
\left(\mathrm{mas} \mathrm{yr}^{-1}\right) \\
\end{array}$ & $\begin{array}{r}p m \operatorname{Dec}^{a} \\
\left(\operatorname{mas~yr}^{-1}\right) \\
\end{array}$ & $\begin{array}{c}G^{a} \\
(\mathrm{mag}) \\
\end{array}$ & $\begin{array}{r}B P-R P^{a} \\
(\mathrm{mag}) \\
\end{array}$ & $\begin{array}{l}\text { Initial } \\
\text { SpT }\end{array}$ & $\begin{array}{l}\text { Adopted } \\
\text { SpT }\end{array}$ & Ref. & $\begin{array}{r}v_{\tan ^{b}}^{b} \\
\left(\mathrm{~km} \mathrm{~s}^{-1}\right) \\
\end{array}$ \\
\hline 171 & LSPM J0937+2803 & 144.359230 & +28.056399 & $3.68 \pm 0.03$ & $+63.38 \pm 0.04$ & $-153.13 \pm 0.04$ & 13.354 & +1.075 & DQ+DAZ? & G-type dC & 1 & 214 \\
\hline 192 & WD 1004+665 & 152.005966 & +66.322420 & $2.19 \pm 0.02$ & $-153.10 \pm 0.03$ & $-127.69 \pm 0.04$ & 14.475 & +0.980 & no WD & $\approx \mathrm{G}$ & 1 & 432 \\
\hline 297 & SDSS J140921.10+370542.6 & 212.337320 & +37.094950 & $2.89 \pm 0.08$ & $-154.80 \pm 0.07$ & $-73.75 \pm 0.09$ & 17.290 & +1.712 & DZ? & M0V (SDSS) & 2 & 282 \\
\hline 308 & LSPM J1445+2527 & 221.414204 & +25.453752 & $1.44 \pm 0.05$ & $-37.82 \pm 0.10$ & $-155.26 \pm 0.10$ & 16.012 & +1.097 & no WD & $\approx \mathrm{G}$ & 1 & 524 \\
\hline 331 & LSPM J1554+1939 & 238.570339 & +19.663440 & $3.47 \pm 0.07$ & $+13.26 \pm 0.09$ & $-179.27 \pm 0.08$ & 16.770 & +1.550 & DC11.0 $\pm 1.0 ?$ & $\approx \mathrm{K}$ & 1 & 246 \\
\hline 351 & 2MASS J16345081+2610073 & 248.711612 & +26.168217 & $1.34 \pm 0.04$ & $-30.34 \pm 0.05$ & $-118.39 \pm 0.07$ & 12.600 & +0.520 & DA6. $0 \pm 1.0 ?$ & $\approx \mathrm{F}$ & 1 & 431 \\
\hline
\end{tabular}

Notes. Gaia DR2 coordinates are for (J2000, epoch 2015.5) and were rounded to 0.000001 degrees, parallaxes and their errors were rounded to

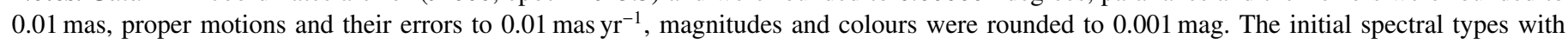
question marks were uncertain because of low $\mathrm{S} / \mathrm{N}$ or possible problems in the flux calibration of the CAFOS spectra. Further notes on the data: ${ }^{(a)}$ Gaia DR2, ${ }^{(b)}$ derived from Gaia DR2 parallaxes and proper motions.

References. (1) This paper adopted spectral types based on CAFOS spectra and the location of the objects along the main sequence in the CMDs (Figs. 9 and 10), (2) West et al. (2011).

with slightly higher spectral resolution of $\sim 1500$, the Balmer line widths of hot DA WDs of lower surface gravity are narrower and may appear similar to those of A-type stars. In a similar way as done by Zhang et al. (2013), we classified objects with blue spectra showing the $\mathrm{Ca} \mathrm{K}$ line in addition to the Balmer lines as A-type (or early F-type) stars. By doing so, we may in principle have excluded some relatively hot DAZ WDs like GD 362 (Gianninas et al. 2004). However, as the Gaia parallaxes have shown (Sect. 5), we do not miss any WD in our classification.

As DA WDs should not show higher-level Balmer lines in lowresolution spectra (Berg et al. 1992), we used their appearance as an additional criterion to classify blue objects with Balmer lines only. All non-DA objects were excluded from further analysis in this study, but are included in Figs. 2 and 3, showing $\mathrm{H} \alpha, \mathrm{H} \beta$, and $\mathrm{H} \gamma$ line width measurements, for comparison.

The spectra of our new and known hot DA WDs, all observed with CAFOS, are presented in Fig. 1. Here we show only those of the known hot DA WDs from our sample that were included in the current census of northern WDs within $40 \mathrm{pc}$ (Limoges et al. 2015) and had consistent spectral types from other sources, too. As one can see, there is only a weak correlation of their spectral types with the slopes of the continuum in our spectra. As the reference spectral types are, according to the errors in effective temperature $(\leqslant 150-300 \mathrm{~K})$, all accurate to within \pm 0.1 subtypes, we assumed that the spectral slopes at the blue end of our spectra are affected by uncertainties in the flux calibration. However, we note that the strongest outlier in the sequence of known objects shown in Fig. 1, CA462 (= WD 2246+223) with a spectral type of DA4.8, appears as blue as the neighbouring objects of much earlier (DA2.4-DA2.8) types and has similar line widths as these objects (see below in Figs. 2 and 3).

Our spectral classification of new hot DA WDs relied on the closest matches with the spectra of known comparison objects. Four spectra, CA037, CA376, CA399, and CA400, are very similar to our hottest comparison DA WD, CA411 (= WD 2032+248; DA2.4). One object, CA082, resembles the DA3.1 WD CA124 (= WD 0809+177) (plotted next to each other in Fig. 1). Because of the weak correlation of known spectral types with the slopes of our spectra, we assigned spectral types in steps of 0.5 subtypes and uncertainties of 1.0 subtypes to these new objects. For the noisy spectrum of CA078, which has the closest match to the DA3.0 spectrum of CA486 (= WD 2329+407), shown on top of Fig. 1, we conservatively assigned an uncertainty of 1.5 subtypes. As a special case with an even larger uncertainty of 2.0 spectral subtypes we consider the relatively blue spectrum, probably caused by an error in the flux calibration, of CA054, which we classified as DA5.0 (also shown in Fig. 4) because of its relatively narrow Balmer lines (see also Figs. 2 and 3).

We measured the equivalent widths of the $\mathrm{H} \alpha, \mathrm{H} \beta$, and $\mathrm{H} \gamma$ lines in our low-resolution spectra of all known $<$ DA7.5 WDs and of the new hot and cool DA WDs (Sect. 4.3) in our sample. In addition, we measured the same three lines in the non-WD spectra classified by us as A-type (or early F-type) stars (see above) 


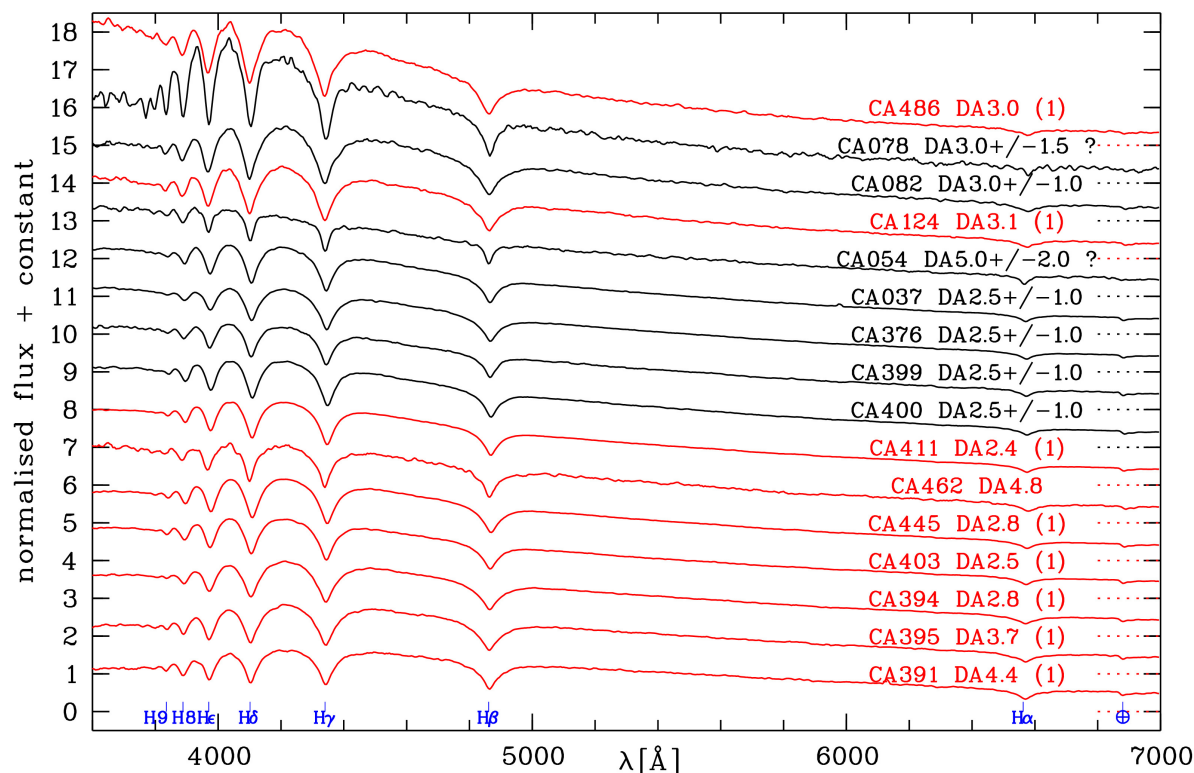

Fig. 1. CAFOS spectra of known (red) and new (black) hot DA WDs. The spectral types of known objects, CA486 =WD 2329+407, CA124 $=$ WD $\quad 0809+\quad 177, \quad$ CA411 $=$ WD $2032+248, \mathrm{CA} 462=\mathrm{WD} 2246+223, \mathrm{CA} 445=$ WD $2149+021, \quad$ CA403 $=$ WD 1943+163, CA394 = WD 1911+536, CA395 = WD 1911+ 135, $\mathrm{CA} 391=\mathrm{GD}$ 532, have uncertainties below \pm 0.1 subtypes according to reference (1) = Limoges et al. (2015). The spectra are roughly sorted by the slope of their continuum. The locations of the Balmer lines and of a terrestrial absorption band are marked on the bottom. and of known sdO and sdB among our comparison objects. The results are presented in Figs. 2 and 3, where one can see a relatively clear separation of three groups of objects: hot DA WDs, cool DA WDs, and non-WDs, in particular for $\mathrm{H} \alpha$ as a function of $\mathrm{H} \beta$. The non-WDs reach equivalent widths of $\mathrm{H} \beta$ up to about $25 \AA$, but show systematically smaller equivalent widths of $\mathrm{H} \alpha$ than the cool DA WDs. The separation between cool DA WDs and non-WDs is less obvious in the $\mathrm{H} \gamma$ vs. $\mathrm{H} \beta$ diagram. The hot DA WDs exhibit large equivalent widths for all three lines, where the maximum values are reached for types around DA4, which are known to have the strongest Balmer lines at normal gravities of $\log g \approx 8$ (Wesemael et al. 1993).

The four new hot DA WDs, CA037, CA376, CA399, and CA400, which were all classified by us as DA2.5 \pm 1.0 based on the closest match of their spectra with that of the known DA2.4 WD CA411 (= WD 2032+248), have equivalent widths of $23 \lesssim \mathrm{H} \alpha \lesssim 31 \AA, 33 \lesssim \mathrm{H} \beta \lesssim 52 \AA$, and $26 \lesssim \mathrm{H} \gamma \lesssim 35 \AA$. These values are similar or slightly smaller compared to those of the two known DA2.4 and DA2.5 WDs shown in Figs. 2 and 3. This is a first indication that the four new DA2.5 WDs may be even slightly hotter than estimated from our initial classification.

One of the new hot DA WDs, CA376 was independently discovered as a wide companion to the well-known nearby star HD 166435 and already spectroscopically classified as DA2.2 \pm 0.2 by Scholz et al. (2018) based on the comparison with only two known WDs, CA411 (= WD 2032+248; DA2.4) and CA445 (= WD 2149+021; DA2.8). This comparison made use of both the spectral slopes and the line widths of $\mathrm{H} \beta$ and $\mathrm{H} \alpha$. Our new spectroscopic classification of CA376 as DA2.5 \pm 1.0 , taking into account the observed spread in the spectral slopes of a larger number of comparison objects, is more conservative but consistent with the former classification.

\subsection{Comparison with model DA WD spectra}

To check our spectral classification of DA WDs, in particular of the hot DA WDs classified in Sect. 4.1, we also compared our observed data with WD model atmosphere spectra kindly made available by D. Koester (Koester 2010). The spectra are for a pure hydrogen atmospheric composition. Balmer lines in the models were calculated with the modified Stark broadening profiles of Tremblay \& Bergeron (2009) kindly made available by these authors to Koester. The models cover the effective temperature range between $6000 \mathrm{~K}$ and $100000 \mathrm{~K}$, and a range in gravity from $\log g=5.00$ to $\log g=9.00$ with a step size of 0.25 dex. Due to the low $\mathrm{S} / \mathrm{N}$ of our obtained spectra, we opted by comparing only equivalent widths to the model spectra, rather than doing a fit. The spectra were normalised with low-order polynomials to selected continuum points, and the equivalent widths of the Balmer lines $(\mathrm{H} \alpha, \mathrm{H} \beta, \mathrm{H} \gamma)$ were measured with the INTEG/LINE command within ESO/MIDAS for both model and observed spectra.

As expected, the model spectra show a general trend to smaller equivalent widths with smaller gravity. However, there are only weak gravity-dependent trends seen for the known DA WDs in our sample, whereas the noise-free model spectra show clear changes in the equivalent widths, according to which for instance the turnoff point caused by the maximum of the Balmer lines changes from about $16000 \mathrm{~K}(\approx \mathrm{DA} 3.2)$ at $\log g=9$ to about $12000 \mathrm{~K}(\approx \mathrm{DA} 4.2)$ at $\log g=7$.

Interestingly, our two DA3.0 WDs CA078 and CA082 are located at two edges of the distributions shown in both Figs. 2 and 3. As the stronger $\mathrm{H} \beta$ and $\mathrm{H} \gamma$ lines in our hot DA spectra are generally better measured than the $\mathrm{H} \alpha$ line, we see less scatter of the observed data around the model data in Fig. 3. In that respect the location of CA078 as a clear outlier, falling in the region of the low-gravity model curve, is remarkable. We will further discuss the possible low gravity status of this uncertain DA3.0 \pm 1.5 candidate in Sect. 7.6.

The better classified DA3.0 $\pm 1.0 \mathrm{CA} 082$ lies close to the turnoff points for the $\log g=9$ model curves in both Figs. 2 and 3 , in good agreement with the effective temperature of about $16800 \mathrm{~K}$. Finally, our very uncertain classification of CA054 as DA5.0 \pm 2.0 is supported by the location of this object in the central regions of both Figs. 2 and 3 at the edges of the areas occupied by hot DA WDs, cool DA WDs, and non-WDs.

\subsection{New cool DA WDs and candidates}

With decreasing effective temperatures the Balmer lines of DA WDs become sharper and weaker. As mentioned by 


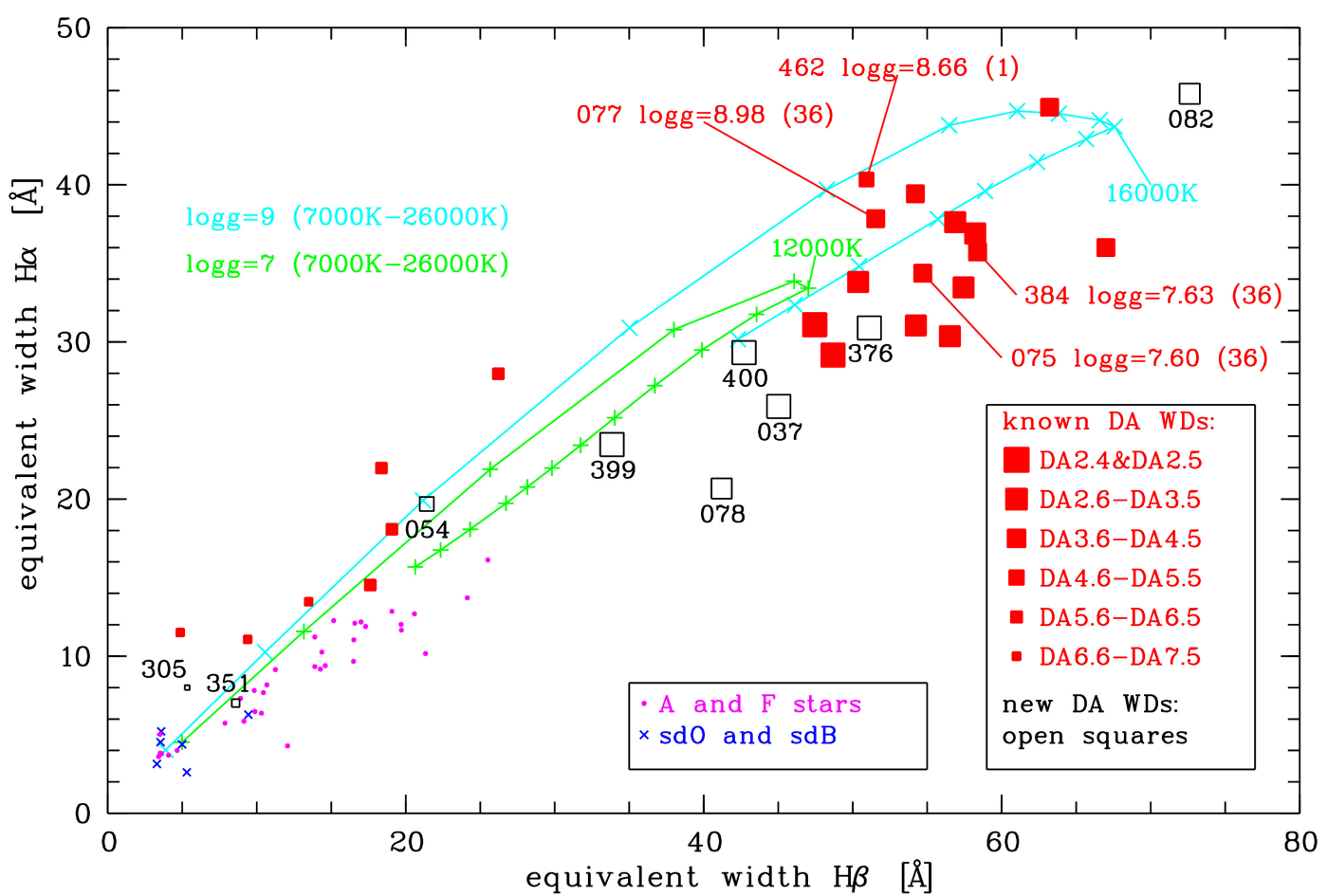

Fig. 2. Equivalent widths of $\mathrm{H} \beta$ and $\mathrm{H} \alpha$ lines measured in the CAFOS spectra of DA WDs and other objects with dominant Balmer lines. The classification of known DA WDs with typical errors of less than about 0.1 subtypes was taken from $(1)=$ Limoges et al. (2015), (2) = Kawka \& Vennes (2012), and (36) = Gianninas et al. (2011). Their gravities, given by these authors, varied between 7.6 $<\log g<9.0$. The objects with the two lowest and highest gravities, CA462 = WD 2246+223, CA384 = WD 1824+040, CA075 = WD 0416+701, CA077 = WD 0457-004, are marked. New DA WDs (and candidates) from Figs. 1 to 4 are also labelled by their CA numbers. The plus signs and crosses connected with solid lines show the measurements of model spectra with $T_{\text {eff }}$ from $7000 \mathrm{~K}$ to $26000 \mathrm{~K}$ (below $20000 \mathrm{~K}$ in steps of $1000 \mathrm{~K}$, above $20000 \mathrm{~K}$ in steps of $2000 \mathrm{~K}$ ) with gravities of $\log g=7$ and $\log g=9$.

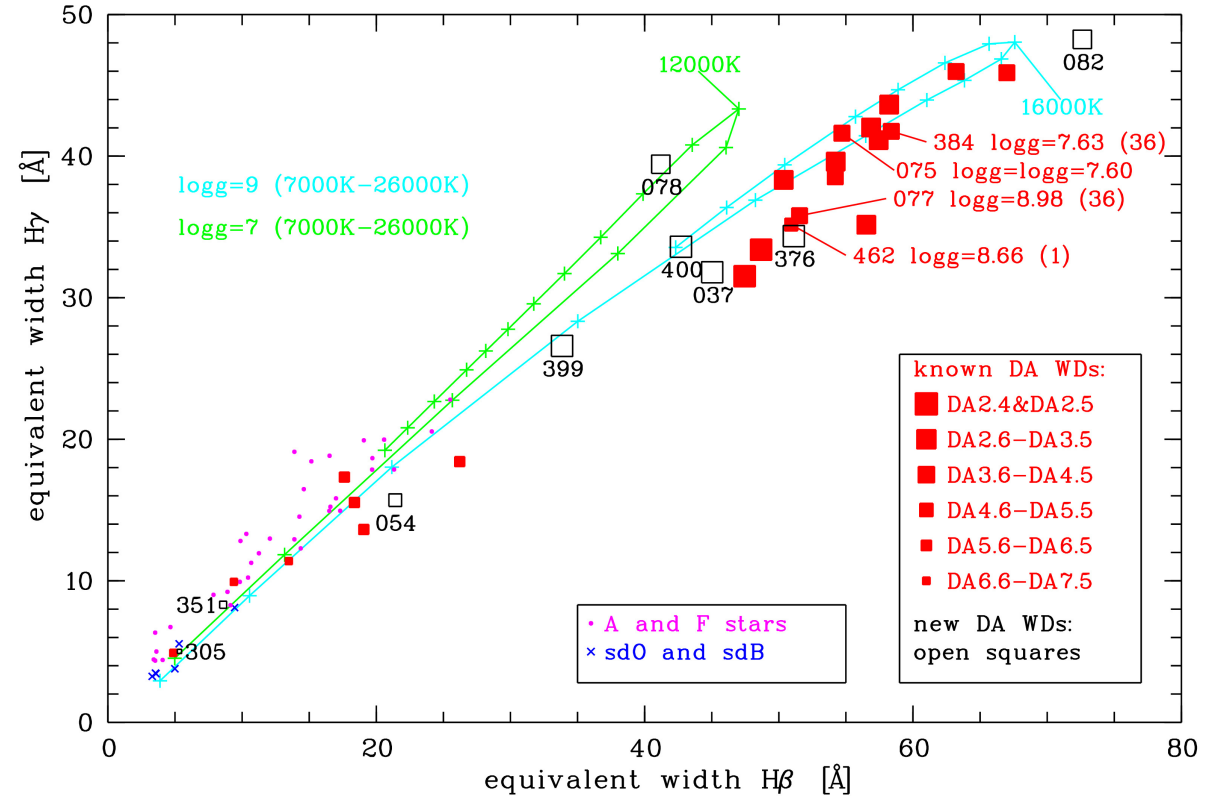

Fig. 3. Same as Fig. 2 for equivalent widths of $\mathrm{H} \beta$ and $\mathrm{H} \gamma$ lines.
Wesemael et al. (1993), at low S/N and with the lowesttemperature DA WDs (beyond DA9) only $\mathrm{H} \alpha$ remains visible, and it becomes harder to distinguish between cool DA and DC WDs (see Sect. 4.4). This can be seen in some of our spectra shown in Figs. 4 and 5.

In Fig. 4 we show our cool DA WD candidates together with an representative sample of known DA5-DA10 WDs. The spectrum of CA054 on top was already discussed in Sects. 4.1 and
4.2 and shown in Fig. 1. The Balmer line widths of this object look similar to those of the known DA5.9 WD CA024 (= WD $0101+048)$, whereas the continuum of CA054 is much bluer. The relatively noisy spectrum of CA351 was classified by us only as a weak DA6 candidate (with question mark), because $\mathrm{H} \alpha$ is not clearly visible, whereas the higher-level Balmer lines appear stronger. The spectrum of CA305 shows the opposite trend of weakening higher-level Balmer lines, also seen for 

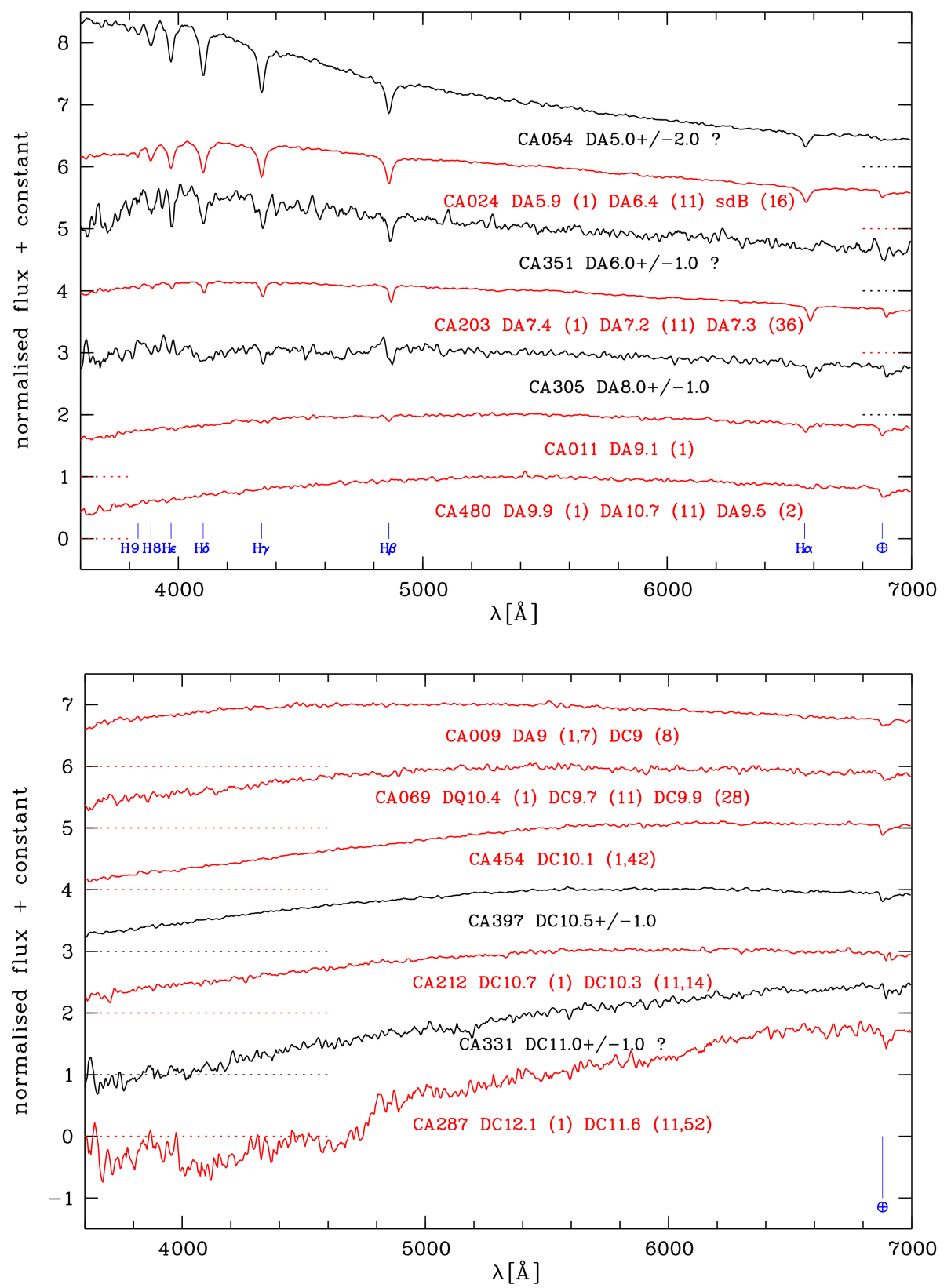

Fig. 4. CAFOS spectra of known (red) and new (black) cool DA WDs. The spectral types of known objects, CA024 = WD 0101+048, CA203 = WD 1019+637, CA011 $=\mathrm{WD} 0025+054, \mathrm{CA} 480=\mathrm{WD} 2322+137$, with typical errors of less than about 0.1 subtypes are taken from the following references: (1) = Limoges et al. (2015), (11) = Sion et al. (2014), (16) = Drilling et al. (2013), and (36) = Gianninas et al. (2011). The spectra are roughly sorted by the slope of their continuum. The locations of the Balmer lines and of a terrestrial absorption band are marked on the bottom.
Fig. 5. CAFOS spectra of known (red) and new (black) cool DC WDs. The spectral types of known objects, CA009 = WD 0019+423, CA069 = WD 0344+014, CA454 = WD $2215+368$, CA2 12 = WD $1033+714$, CA287 $=$ LSPM J1341+0500, with typical errors of less than about 0.1 subtypes are taken from the following references: $(1)=$ Limoges et al. (2015), (7) = Putney (1997), (8) = Greenstein $(1984),(11)=$ Sion et al. (2014), (14) = Holberg et al. (2008), (28) = Subasavage et al. (2007), (42) = Giammichele et al. (2012), and (52) = Sayres et al. (2012). The spectra are roughly sorted by the slope of their continuum. A terrestrial absorption band is marked. the coolest known DA WDs at the bottom of Fig. 4. Taking into account the slightly different spectral classification of the comparison objects and the moderate trend towards a bluer continuum with decreasing temperature, we assigned again uncertainties of 1.0 spectral subtypes to our new cool DA candidates.

The classification of CA024 (= WD 0101+048) as sdB (see Fig. 4) seems to be a mismatch in SIMBAD. Drilling et al. (2013) used the wrong name PG $0101+040$ for the sdB PG $0101+039$, and SIMBAD apparently by mistake associated this sdB to PG $0101+048$, which is in fact the known DA WD CA024 as confirmed with the first two and more recent classifications by Limoges et al. (2015) and Sion et al. (2014) given in Fig. 4.

\subsection{New cool DC WDs and candidates}

Featureless spectra, traditionally defined as showing no line deeper than $5 \%$ of the continuum, belong to the class of DC WDs
(Wesemael et al. 1993). Our low-resolution spectra allow only for a first selection of DC candidates. With higher S/N observations weak features can be detected in many WDs initially classified as DC, but true DC WDs do still exist, especially at lower effective temperatures (Wesemael et al. 1993). Therefore, we expected to find in particular faint DC candidates in our target list. Whereas about two thirds of the 226 WDs in the $25 \mathrm{pc}$ sample of Holberg et al. (2016) are DA WDs, the DC WDs represent the second largest fraction with $\approx 17 \%$.

Among the featureless spectra of known and candidate DC WDs shown in Fig. 5, the coolest known DC WD CA287 $(\approx \mathrm{DC} 12)$ and the coolest new candidate CA331 were observed with only low S/N. Consequently, we consider CA331 as doubtful DC11 candidate (with question mark). Our second new DC WD, CA397, shows a very smooth featureless spectrum and was classified as DC10.5 \pm 1.0 based on its comparison with the known DC9-DC11 WDs observed by us. With our assigned uncertainty of 1.0 spectral subtypes we again took into account the slight variations in the slopes of the spectra of comparison 

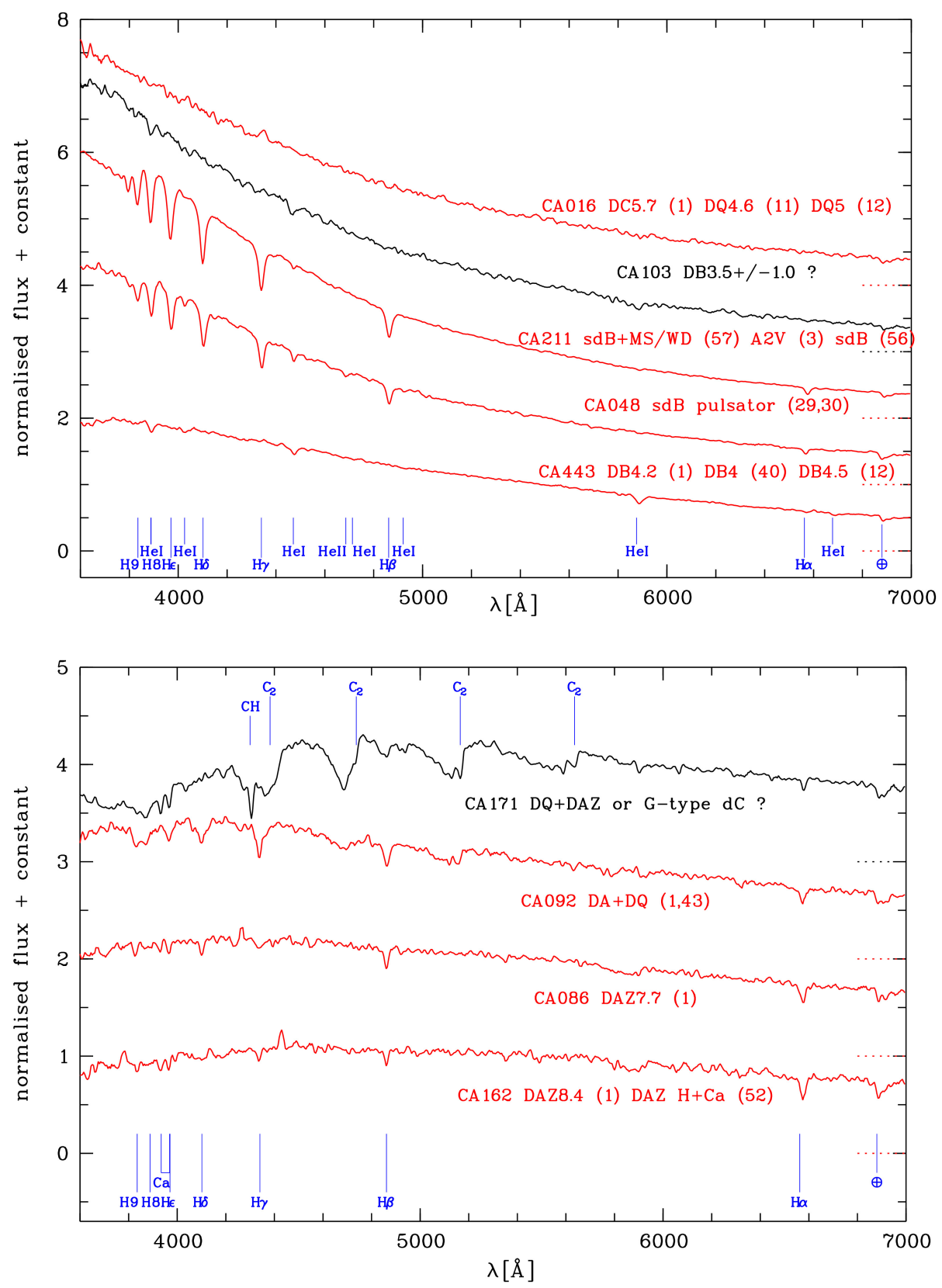

Fig. 6. CAFOS spectra of known (red) cool DB and DC WDs and sdB stars, CA016 = WD $0038+555$, CA $211=$ PG 1032+406, CA048 $=\mathrm{GD} 1053$, CA443 = WD $2147+280$, and of a new (black) cool DB candidate. The spectral types of known objects with typical errors of less than about 0.1 subtypes are taken from the following references: $(1)=$ Limoges et al. (2015), (3) = Luo et al. (2015), $(11)=$ Sion et al. (2014), (12) $=$ Holberg et al. (2003), (29) = Randall et al. (2014), (30) = Reed et al. (2012), (56) = Østensen et al. (2010), and (57) = Kupfer et al. (2015). The spectra are roughly sorted by the slope of their continuum. The Balmer lines, He I and He II lines, and a terrestrial absorption band are marked on the bottom.
Fig. 7. CAFOS spectra of known (red) DAZ and DA+DQ WDs, CA092 = GD 73, CA086 = LSPM J0543+3637, CA162 = WD $0920+012$, and of a new DQ+DAZ or G-type $\mathrm{dC}$ candidate (black). The spectral types of known objects with typical errors of less than about 0.1 subtypes are taken from the following references: (1) = Limoges et al. (2015), (43) = Vennes \& Kawka (2012), and (52) = Sayres et al. (2012). Locations of the Balmer lines, the $\mathrm{Ca} \mathrm{H}$ and $\mathrm{K}$ lines, and of a terrestrial absorption band are marked on the bottom. The bandheads of four $\mathrm{C}_{2}$ Swan bands and the location of the $\mathrm{CH}$ band are marked on top. objects as well as the range of their spectral types given by different authors.

\subsection{A new $D B W D$}

Those WDs that show only helium lines in their spectra are called DO (with effective temperatures generally above $45000 \mathrm{~K}$ and He II lines) or DB (with lower temperatures, corresponding to the range DB2-DB5, and only He I lines; Wesemael et al. 1993). There are only two DB WDs among the 226 WDs in the $25 \mathrm{pc}$ sample of Holberg et al. (2016). Therefore, we had only one known DB WD (CA443 = WD 2147+280) selected as a comparison object in our target list. Nevertheless, we were able to identify one new DB candidate (CA103) in our spectroscopic data set. The spectra of both objects are shown in Fig. 6 together with the spectra of two known sdB stars and a known DC WD.

Concerning the spectra and marked absorption lines in Fig. 6, we note that the $\mathrm{H} 8$ line of hydrogen and the He I line at $3889 \AA$ are located very close to each other (Stellmacher et al. 1992). While we see the H8 line as part of the dominating Balmer line series in the spectra of the two hot subdwarfs shown in Fig. 6 for comparison, we can see the He I line at $3889 \AA$ together with two other He I lines (at $4471 \AA$ and $5876 \AA$ ) in the spectra of both the known $\approx$ DB4 WD $(C A 443=$ WD $2147+280)$ and of our new DB3.5 candidate CA103. A featureless spectrum of a known $\approx$ DC6/DQ5 WD is shown on top to demonstrate the weakness of the He I lines in the spectra of the DB WDs. As one can see in Fig. 7 of Wesemael et al. (1993), the classical DB WDs exhibit more He I lines at higher temperatures (DB2) than at the cool end of the DB sequence (DB5), where only few He I lines remain visible, including those at $3889 \AA$ and $4471 \AA$. This led us classify CA103 as a not much earlier type than CA443 (= WD 2147+280), despite of the rather different slopes of their continuum, with an uncertainty of one spectral subclass and a question mark because of the weakness of the spectral features with respect to the noise. 


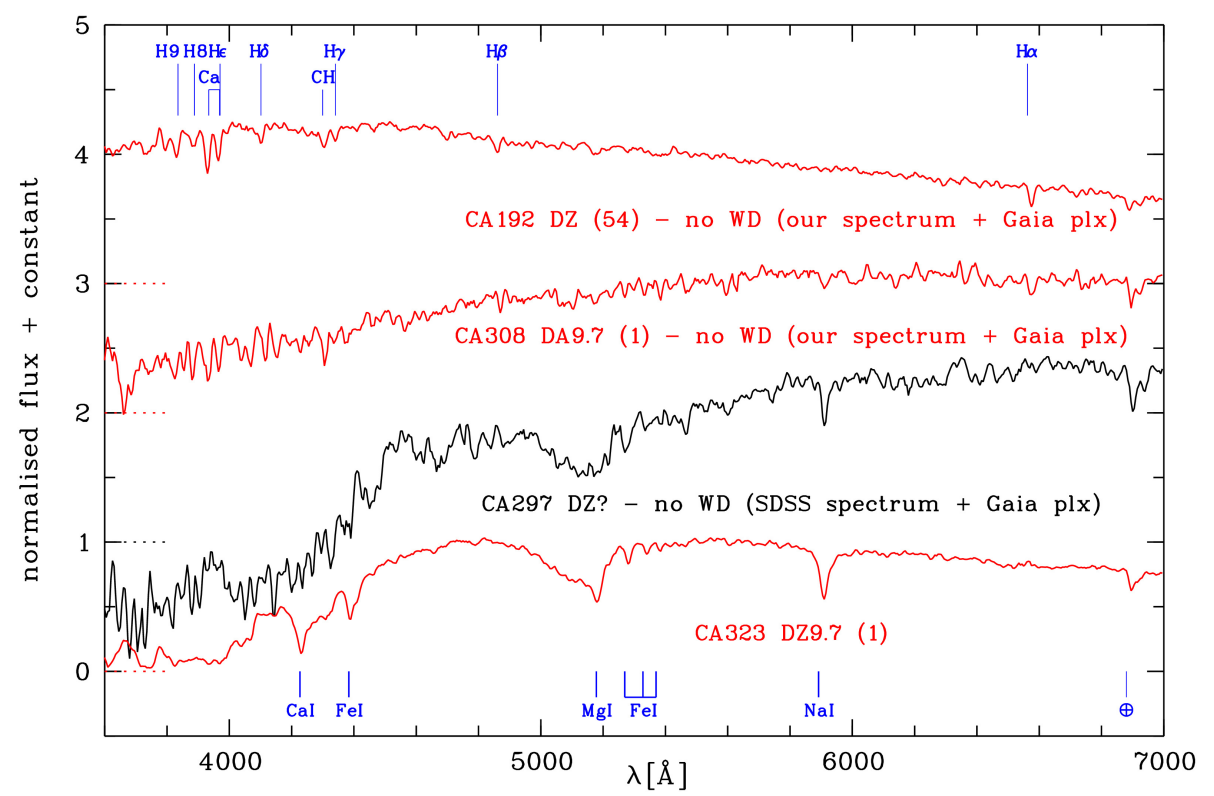

Fig. 8. CAFOS spectra of two rejected known WDs (plotted in red on top), CA192 = WD 1004+665, CA308 = LSPM J1445+2527, a rejected new DZ candidate (black), and a known DZ WD. CA323 = WD 1532+129, (plotted in red on bottom). The spectral types of known objects with typical errors of less than about 0.1 subtypes are taken from the following references: $(1)=$ Limoges et al. (2015) and (54) $=$ Sayres et al. (2012). Locations of the strongest metallic lines in the spectrum of the known DZ WD and of a terrestrial absorption band are marked on the bottom. Those of the Balmer lines, the $\mathrm{Ca} \mathrm{H}$ and $\mathrm{K}$ lines, and the $\mathrm{CH}$ band are marked on top.

\subsection{A new $D Q+D A Z W D$ or G-type carbon dwarf?}

The third largest fraction $(\approx 11 \%)$ of WDs in the $25 \mathrm{pc}$ sample (Holberg et al. 2016) is formed by 24 DQ WDs with atomic or molecular carbon features in their spectra. This fraction is followed by ten DZ WDs $(\approx 4 \%)$, which show mainly other metallic lines ( $\mathrm{Ca}, \mathrm{Mg}, \mathrm{Fe}, \mathrm{Na}$ ). Another ten WDs in the $25 \mathrm{pc}$ sample form a sub-group in the DA WD fraction, the so-called DAZ WDs with strong Balmer lines and weak metal lines.

Among our spectra we found one peculiar WD candidate CA171 with characteristic spectral features of several WD classes. It resembles partly that of the known DA+DQ close binary CA092 (= GD 73; Vennes et al. 2012; Fig. 7). The spectrum of CA171 shows as most prominent features all four Swan bands (at $4382 \AA, 4736 \AA, 5165 \AA$, and $5635 \AA$ ) of molecular carbon typically seen in DQ WDs (Wesemael et al. 1993) but also the $G$ band of $\mathrm{CH}$ at $4300 \AA$. In that respect, it looks very similar to the peculiar DQP8 WD G 99-37 (=WD 0548-001) in Fig. 19 of Wesemael et al. (1993). However, the spectrum of CA171 shows as additional features the $\mathrm{H} \alpha$ (and $\mathrm{H} \beta$ ) and the $\mathrm{Ca}$ $\mathrm{H}$ and $\mathrm{K}$ lines, typical for DAZ WDs, two of which are shown for comparison in the lower part of Fig. 7. Therefore, we considered CA171 as a DQ+DAZ binary WD candidate.

Our alternative classification of this relatively bright $(G \approx$ $13.35 \mathrm{mag})$ and moderately blue $\left(G-J \approx+1.27 \mathrm{mag}, J-K_{\mathrm{s}} \approx\right.$ $+0.42 \mathrm{mag})$ high proper motion $\left(\approx 166 \mathrm{mas} \mathrm{yr}^{-1}\right)$ star is that of a so-called G-type carbon dwarf $(\mathrm{dC})$ star. This class of objects was investigated on the basis of spectroscopic data from the SDSS by Green (2013). An example spectrum is presented in his Fig. 2, which exhibits the same main spectral features as observed by us with lower resolution and $\mathrm{S} / \mathrm{N}$ for CA171.

\subsection{Other spectroscopically rejected (candidate) WDs}

During our visual classification by comparing the spectra of new WD candidates with those of known WDs, we mentioned two previously known WDs, CA192 (= WD 1004+665) and CA308 (= LSPM J1445+2527), that failed our own WD classification. In their spectra shown in the upper part of Fig. 8 we identified weak Balmer lines in addition to the $\mathrm{Ca} \mathrm{H}$ and $\mathrm{K}$ lines as well as the $\mathrm{G}$ band of $\mathrm{CH}$ (all lines are marked on top of Fig. 8). Therefore, we classified these objects as F- or G-type stars.

The spectrum of our faintest $(G \approx 17.3 \mathrm{mag})$ object CA297 turned out to be peculiar and relatively red among our targets. We found the known DZ9.7 WD CA323 $(=\mathrm{G} 137-24=$ WD 1532+129) as its closest match. According to Hollands et al. (2017) this is the nearest known DZ WD.

The most prominent lines of CA323 as listed in Kawka et al. (2004) are marked at the bottom of Fig. 8. The very asymmetric profile of the most prominent $\mathrm{Mg}$ I line at $5170 \AA$ was mentioned in this paper. Adopting the explanation by Wehrse \& Liebert (1980) this is the result of quasi-static van der Waals broadening. This broad feature, as well as some other characteristic lines ( Na I and Fe I) in the spectrum of CA323 seem also to be present in the spectrum of CA297.

However, in Favia et al. (2015) CA297 (=SDSS $\mathrm{J} 140921.10+370542.6$ ) is listed as a runaway $\mathrm{M}$ dwarf candidate. Its SDSS spectrum is of higher $\mathrm{S} / \mathrm{N}$ than our CAFOS spectrum and clearly rules out a WD. It was classified as that of a K7 (SDSS DR14 spectroscopic data base) or M0V star (SIMBAD reference: West et al. 2011).

\section{Absolute magnitudes from Gaia DR2 parallaxes}

Almost all of our 410 blue proper motion stars have measured parallaxes in Gaia DR2 (Gaia Collaboration 2018b). The four stars that are lacking Gaia DR2 parallaxes were all classified by us (and in the literature) as non-WDs. In Figs. 9 and 10 we show optical-to-NIR and optical CMDs, where the absolute Gaia magnitudes on the $y$-axis were computed from Gaia DR2 magnitudes and parallaxes. The optical-to-NIR colours shown in Fig. 9 were determined from the observed Gaia DR2 $G$ magnitudes and 2MASS $J$ magnitudes of our targets, whereas the $x$-axis in Fig. 10 represents their Gaia DR2 $B P-R P$ colours. We note that the majority of our spectroscopic targets (shown by green dots) is dominated by subdwarfs rather than normal dwarf stars (compare with the full Gaia DR2 $100 \mathrm{pc}$ sample shown in Fig. 10 by yellow dots), because of our combined proper motion and colour selection (Sect. 2). 


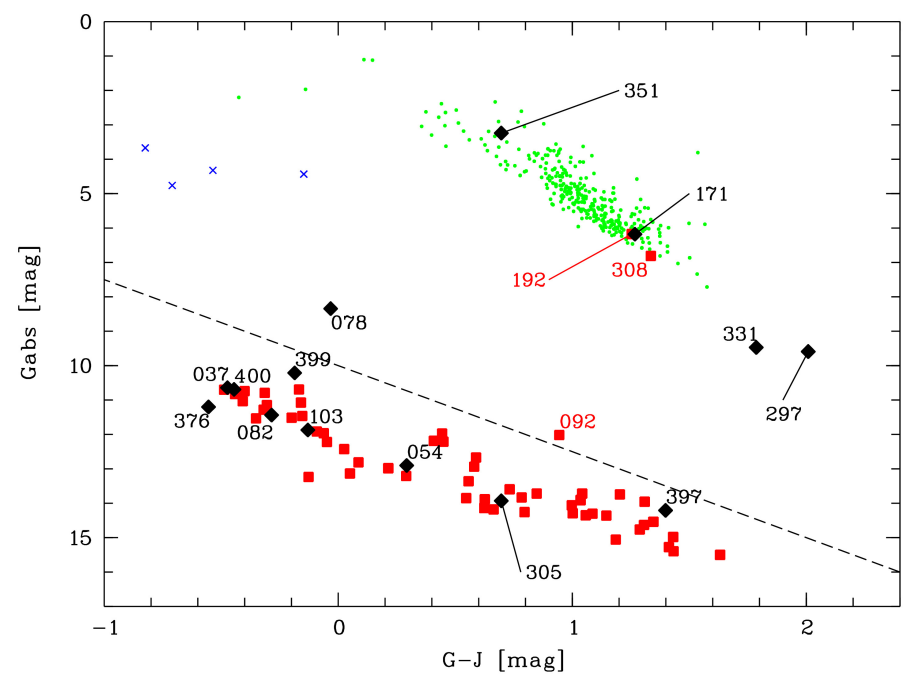

Fig. 9. Optical-to-NIR (Gaia DR2 - 2MASS) CMD of the objects in our spectroscopic sample: red squares $=$ previously known WDs (including two rejected), black lozenges $=$ new WD candidates (confirmed and rejected), blue crosses $=$ hot subdwarfs $(\mathrm{sdO}$ and $\mathrm{sdB})$, green dots $=$ all remaining stars (mainly representing FGK subdwarfs). New WDs and candidates, rejected known WDs, and one known WD binary are marked by their CA numbers. The dashed line corresponds to one of two colour-magnitude limits for WDs used in Scholz et al. (2018).

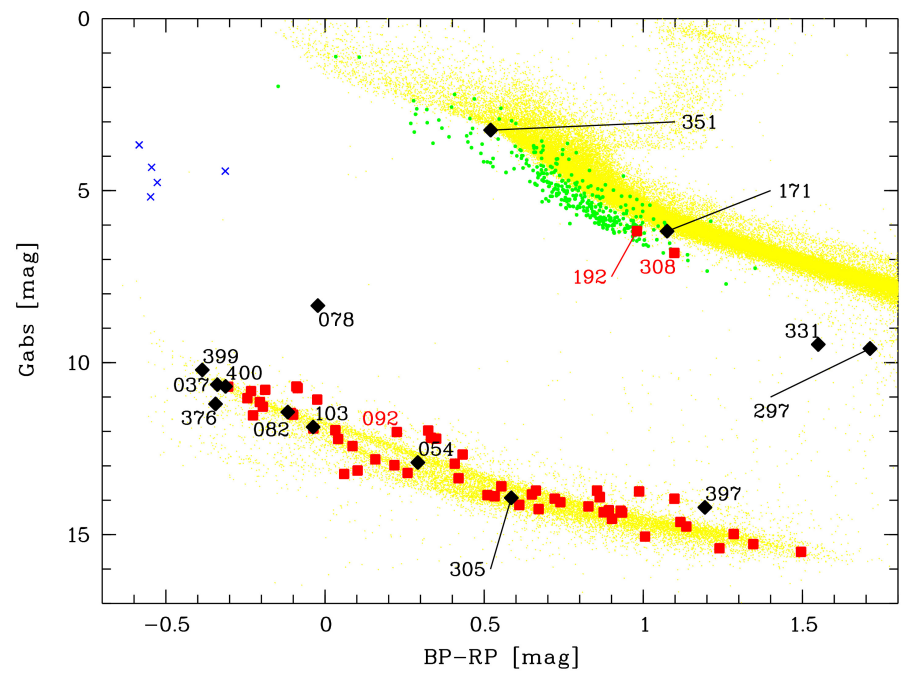

Fig. 10. Optical (Gaia DR2) CMD of the same objects and with the same symbols as in Fig. 9 Yellow dots in the background show all of the 242582 objects of the high-quality Gaia DR2 100 pc sample (Lindegren et al. 2018) that fall in this region of the CMD.

All previously known WDs, except for two objects (CA192 and CA308) already found to be non-WDs according to our spectroscopic classification (Sect. 4.7), occupy the lower left part of Fig. 9 as expected for WDs. Compared to the WD colourmagnitude limit used in the study of Scholz et al. (2018) based on parallaxes and $G$ magnitudes from the Tycho-Gaia Astrometric Solution (TGAS) of Gaia DR1 (dashed line in Fig. 9), only one known WD (CA092 with our observed spectrum shown in Fig. 7) lies slightly above this line. This was not unexpected since CA092 (= GD 73) is known as a close WD binary (Vennes et al. 2012). However, in the optical CMD in Fig. 10 it does neither appear over-luminous nor too red compared to other known WDs in our sample.

Concerning our new WDs and WD candidates, the weak DA6 candidate CA351 (Sect. 4.3), the doubtful DC11 candidate
CA331 (Sect. 4.4), the DQ+DAZ binary WD candidate CA171 (Sect. 4.6), and our DZ candidate CA297 with an SDSS spectrum that already ruled out its WD nature (Sect. 4.7) are clearly rejected as WDs by their Gaia DR2 parallaxes and fall in the subdwarf sequences of Figs. 9 and 10. This is in particular interesting for CA171, which we alternatively classified as a G-type carbon dwarf (Sect. 4.6). Green (2013) noted that G-type dC stars appear bluer than similar mass main sequence stars because of the absorption bands of molecular carbon. They estimated their absolute $i$-band magnitudes as $M_{i} \approx 7.25 \mathrm{mag}$, similar to those of K7-M0 dwarfs. This is consistent with the $G \approx 6.2 \mathrm{mag}$ of CA171.

For all other new WDs and most of the remaining uncertain candidates including CA054, the WD candidate with the largest uncertainty in our spectral classification (DA5.0 \pm 2.0 ) and our DB3.5 candidate CA103, the Gaia DR2 parallaxes fully confirm their WD status. These new WDs are located along the WD sequences in both Figs. 9 and 10, with a concentration at the blue end, especially in the optical CMD (Fig. 10).

One new WD candidate, CA078 (with our uncertain spectral classification of DA3.0 \pm 1.5 ), deserves special attention. It is located about $3 \mathrm{mag}$ above the WD sequence at about zero colour indices (also typical of A0 stars) in both Figs. 9 and 10. We checked the Gaia DR2 data of this object concerning astrometric and photometric quality flags as described in Lindegren et al. (2018). We found that CA078 with a highly significant Gaia DR2 parallax of $13.97 \pm 0.05$ mas and very large $B P$ and $R P$ mean flux over error ratios $(\approx 459$ and $\approx 763)$ falls well in their "Selection A", which represents a basic 100 pc sample. Its astrometric excess noise is small ( 0.145 mas). However, CA078 has a magnitude of $G \approx 12.6 \mathrm{mag}$, and the behaviour of the astrometric excess noise may be less discriminating at $G \lesssim 15$ mag according to Lindegren et al. (2018). The alternative unit weight error $u$ used by these authors for their second "Selection B" is based on other parameters characterising the quality of the astrometric solution. It can be computed for CA078 as $u \approx 2.27$, which is still below the limit of $u \approx 5$ at the given $G$ magnitude (see Fig. C.2 in Lindegren et al. 2018). Their final and third criterion concerning the flux excess factor was taken from Gaia Collaboration (2018a) and led to their "Selection C". As this last criterion mainly concerns photometric problems of faint objects in crowded fields it poses no problems for CA078.

As a member of the high quality $100 \mathrm{pc}$ sample corresponding to Selection C of Lindegren et al. (2018), the clearly overluminous WD CA078 can also be seen in their cleaned CMD (their Fig. C.1, right panel) as one of very few objects in the region between the main sequence and the WD sequence. In fact, it appears there as one of only two objects with colour indices $B P-R P \approx 0.0 \mathrm{mag}$ and 3-4 mag above the WD sequence, but also 3-4 mag below a small group of five possible A-type subdwarfs located about 2 mag below the main sequence, in their cleaned CMD (yellow dots in our Fig. 10). This is a remarkable uncommon object found among our ten spectroscopically confirmed new WDs in a small sample of 410 blue proper motions stars and the much larger Selection $\mathrm{C}$ sample of Lindegren et al. (2018) consisting of 242582 objects. We further discuss the possible binary status of CA078 in Sect. 7.6.

\section{WD classification from Gaia DR2 colours}

To further improve the classification of our new WDs, we investigated the effective temperatures available from the literature 


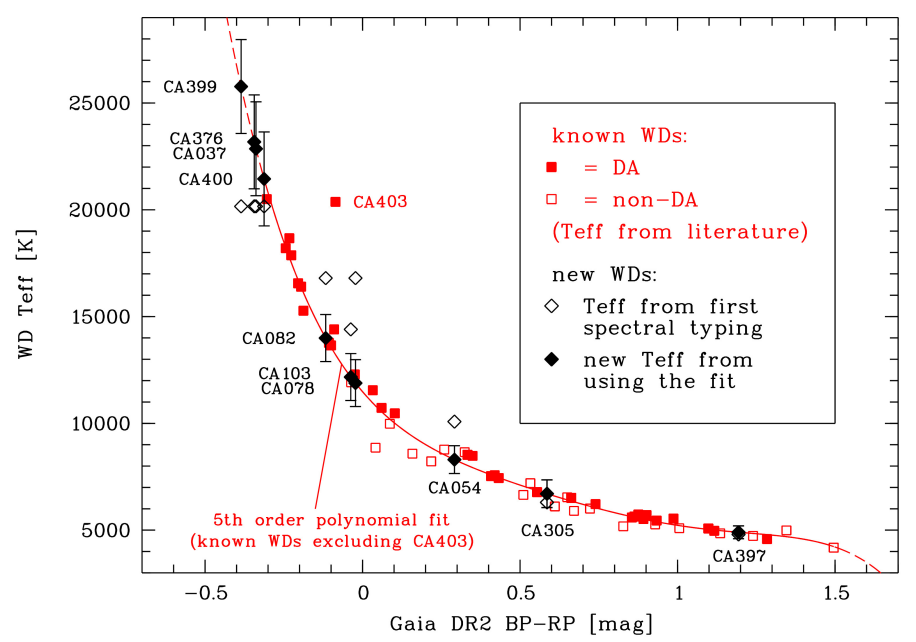

Fig. 11. WD effective temperatures (from literature) as a function of Gaia colours $B P-R P$ for known (and confirmed) DA (filled red squares) and non-DA (open red squares) WDs. The solid and dashed red lines show the 5th order polynomial fit for these objects excluding CA403 (=WD 1943+163; see text) and its extrapolations. Black open lozenges mark the effective temperatures of new (and confirmed) WDs corresponding to their initial spectroscopic classification (Sect. 4) as a function of their Gaia colours. Black filled lozenges mark their newly derived effective temperatures using the fit with known WDs (error bars are explained in the text). The fitted values of new WDs are marked by their CA names.

of all known (and confirmed by Gaia DR2 parallaxes) WDs in our sample as a function of the measured Gaia DR2 colours $B P-R P$ and found a strong correlation between these two quantities (red filled and open squares in Fig. 11). The effective temperatures were mainly taken from Limoges et al. (2015), with additional data from Gianninas et al. (2011), Sion et al. (2014) Kawka \& Vennes (2012), and Luo et al. (2015). The binary WD CA092 was not included here, as its effective temperature was lacking. The red solid line shows a 5th order polynomial fitting these data of the previously known WDs. Excluded from the fit was the WD CA403 (=WD 1943+163), which is a clear outlier. Among all known WDs in our sample it is the only object with a Gaia duplicated source flag (=1). According to Gaia DR2 documentation, such a flag may indicate "observational, cross-matching or processing problems, or stellar multiplicity, and probable astrometric or photometric problems in all cases".

The filled black lozenges show the newly determined effective temperatures of our new WDs applying this polynomial fit, where we had to extrapolate at the hot end (dashed line). Their initially estimated rough effective temperatures according to our former spectroscopic classification are shown by open black lozenges. The maximum deviations of the known WDs (red filled and open squares) from the fitted curve amount to $\pm 1100 \mathrm{~K}$ in the colour interval $(-0.3 \lesssim B P-R P \lesssim 0.0) \mathrm{mag}$, where the curve is steeper, but only $\pm 650 \mathrm{~K}$ in the colour interval $(+0.2 \lesssim B P-R P \lesssim$ $+0.9) \mathrm{mag}$ and $\pm 300 \mathrm{~K}$ in the colour interval $(+0.9 \lesssim B P-R P \lesssim$ $+1.5) \mathrm{mag}$, where the curve is more and more flattening. We conservatively estimated the uncertainties of our newly determined effective temperatures for new WDs to be as large as these maximum deviations in the corresponding colour intervals, but applied an additional factor of 2.0 for the very blue $(-0.4 \lesssim B P-R P \lesssim$ -0.3) mag colours, where we had to extrapolate.

The resulting uncertainties in effective temperatures yield also smaller errors in the determination of the spectral type from \pm 0.2 subtypes for our new hot WDs to about \pm 0.8 subtypes for our new cool WDs. Our initial spectral types and our finally adopted spectral types of higher precision agree within their assigned uncertainties (Table 1).

\section{Discussion}

\subsection{Classification and kinematics of rejected WDs}

Three objects (CA171, CA308, and CA351) out of six rejected WD candidates and known WDs described in Sects. 4.6 and 4.7 were photometrically classified as FGK stars by Pickles \& Depagne (2010), and one object (CA192) was listed as $\mathrm{K}$ star candidate in the catalogue of Kharchenko (2001). The WD classification of CA297 was rejected on the basis of an SDSS M0V spectrum already noted in Sect. 4.7.

The largest tangential velocity among our new WDs is $77 \mathrm{~km} \mathrm{~s}^{-1}$ for CA399, possibly indicating its membership in the Galactic thick disk population. On the other hand, all rejected WDs exhibit tangential velocities between $\approx 210 \mathrm{~km} \mathrm{~s}^{-1}$ and $\approx 520 \mathrm{~km} \mathrm{~s}^{-1}$, relating them to the Galactic halo. They are all located at high Galactic latitudes (between $+40^{\circ}$ and $+71^{\circ}$ ), whereas the ten new WDs lie near the Galactic plane between $-29^{\circ}$ and $+40^{\circ}$.

The Gaia parallax of CA171 (Table 2) confirms its classification as a G-type carbon dwarf. With the corresponding distance of $\approx 272 \mathrm{pc}$ it is a relatively nearby representative of this class of objects. The vast majority of G-type dC stars presented by Green (2013) are much fainter than CA171 and show typical total proper motions below $30 \mathrm{mas} \mathrm{yr}^{-1}$ (see his Fig. 11). Compared to other nearby carbon dwarfs (Harris et al. 2018), distance and tangential velocity of CA171 are typical for the Galactic halo population. But, compared to other carbon dwarfs it appears much bluer $\left(J-K_{\mathrm{s}} \approx+0.4 \mathrm{mag}\right)$ and relatively bright $(J \approx 12.1 \mathrm{mag})$ not only in the NIR (2MASS) but also in the optical ( $B=14.59 \mathrm{mag}, V=13.55 \mathrm{mag}$ ) (APASS; Henden et al. 2016).

\subsection{Previous classification of new WDs}

Three of the new WDs, CA037 (=GD 277), CA054 (=GD 28), and CA399 (=GD 221), were already considered as WD candidates by Giclas et al. (1980). Later on two of them were classified as non-WDs in Pickles \& Depagne (2010) based on multi-colour photometry (CA037 as A7III candidate and CA054 as G8V candidate). Another new WD, CA397, was previously listed as a Kepler target star with estimated 1.5 solar masses and about solar metallicity (Huber et al. 2014). Two of the new WDs, CA054 and CA305 were listed in Luyten's WD catalogue (Luyten 1999), both with a spectral or colour class "f".

\subsection{URAT parallaxes of new WDs}

For four of the new WDs (CA037, CA103, CA376, and CA397) first trigonometric parallaxes of lower precision were already published before Gaia DR2 in the URAT (Zacharias et al. 2015) Parallax Catalog (UPC; Finch \& Zacharias 2016b, 2018) described by Finch \& Zacharias (2016a), Finch et al. (2018). For two of them, CA037 and CA103, the UPC parallaxes are in excellent agreement with the Gaia DR2 values, and for CA397 the UPC parallax agrees within the typical UPC error bars ( $\pm 3-6$ mas) with the Gaia DR2 parallax. Only for CA376, 
the UPC parallax is about 10 mas larger than the Gaia DR2 parallax.

\subsection{Wide binary companions of our new WDs}

Wide binaries were traditionally found among common proper motion stars in high proper motion catalogues. Now, in the Gaia era, we can use very accurate measurements of both proper motions and parallaxes for an almost complete sample of stars in the solar neighbourhood. Therefore, we checked our ten new WDs for stars with common proper motion and parallax in Gaia DR2 data. We used a search radius of 3600 arcsec and selected only Gaia DR2 sources with very similar parallaxes and then compared their proper motions.

In addition to the already known G-type primary of CA376 (=HD 166435 B; see Scholz et al. 2018) with an angular separation of about 29.2 arcsec, we found 2MASS J19293859+1118050 a red $(B P-R P \approx+2.62 \mathrm{mag})$ companion of CA400 separated by only about 12.7 arcsec. Its parallax $(27.38 \pm 0.07$ mas $)$ is in perfect agreement with that of CA400, whereas its proper motion of $(+18.76 \pm 0.11,99.80 \pm 0.09)$ mas $\mathrm{yr}^{-1}$ is only slightly deviating (Table 1), as expected due to orbital motion (Scholz et al. 2018). We derived an absolute $J$ magnitude of $\approx 7.3$ mag for this red companion from its 2MASS photometry and Gaia DR2 parallax corresponding to a spectral type of $\approx \mathrm{M} 3$ according to the relationship between absolute magnitudes and spectral types (Scholz et al. 2005).

\subsection{One of the nearest DB WDs: $C A 103$}

With a distance of only $\approx 26.60 \mathrm{pc}, \mathrm{CA} 103$ is the second nearest of our new WDs after CA376 at $\approx 24.45$ pc (based on Gaia DR2 parallaxes). There were only two DB WDs in the $25 \mathrm{pc}$ WD sample (Holberg et al. 2016), and one of these two, WD 2058+342, is now found to be at a distance of $\approx 52.51 \mathrm{pc}$ according to its Gaia DR2 parallax ${ }^{3}$. Therefore, CA103 may in fact be the second nearest of all DB WDs after WD 1917-074 at $\approx 10.50 \mathrm{pc}$.

Among our new WDs (Table 1), CA103 has a very small proper motion for its distance, and consequently the smallest tangential velocity of $\approx 10 \mathrm{~km} \mathrm{~s}^{-1}$, indicating a young age. Interestingly, CA103 is listed in the K2 Ecliptic Plane Input Catalog (EPIC; Huber \& Bryson 2017). It represents a relatively bright $(G \approx 13.9 \mathrm{mag}, J \approx 14.0 \mathrm{mag})$ target for various follow-up observations.

\subsection{CA078, a nearby extremely low mass WD candidate}

The multiplicity of nearby WDs, in particular double WDs (DWDs) and WD main-sequence binaries (WDMS), was investigated by Toonen et al. (2017). They listed two unresolved DWDs and eight unresolved DWD candidates within $25 \mathrm{pc}$ (mostly DA types between DA5.6 and DA9.9) in their Table 1. In addition, one of their only four resolved DWDs (DC10) has a small angular separation of 1.4 arcsec. They also compared the observed multiplicity of WDs with star formation and evolution model predictions and found a discrepancy for resolved DWDs, more than ten of these are apparently missing within $20 \mathrm{pc}$.

Our overluminous WD CA078 lies at a much larger distance of about $71.6 \mathrm{pc}$, where the chances to resolve a possible mul-

\footnotetext{
3 Investigating the Gaia DR2 20 pc sample, Hollands et al. (2018) idendified 128 known and 11 new WDs, whereas 57 (!) former members of the $20 \mathrm{pc}$ sample were found to be located at larger distances.
}

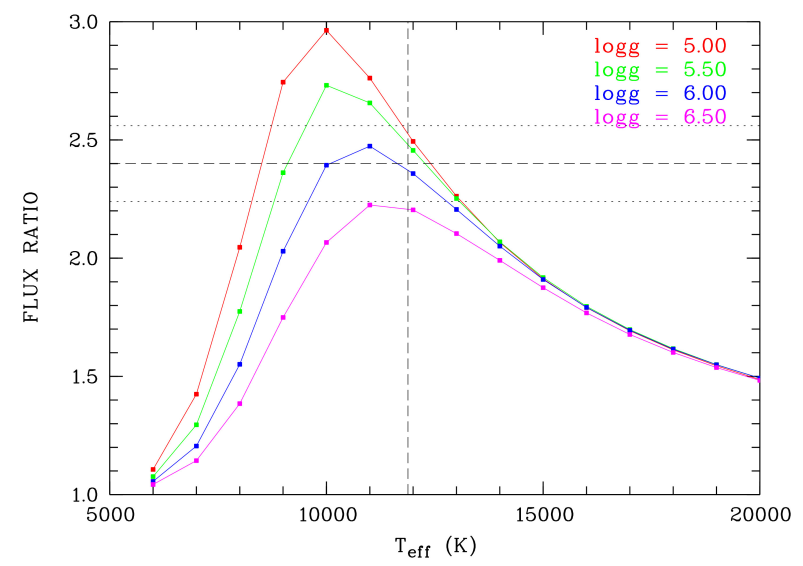

Fig. 12. Balmer jump as a function of effective temperature measured in low-gravity model spectra (small filled squares connected by solid lines) between $\log g=5.0$ (top) and $\log g=6.5$ (bottom). The dashed vertical line indicates the effective temperature of CA078 (Sect. 6). Dashed and dotted horizontal lines indicate our observed value for CA078 and our estimated measurement uncertainty.

tiple system are lower, and has a slightly earlier spectral type (DA4.2 \pm 0.5 ) than the above mentioned unresolved DWDs. In addition, an equal-mass WD binary would appear only $0.75 \mathrm{mag}$ brighter than a single WD. As already mentioned in Sect. 5, CA078 belongs to the high-quality Gaia DR2 100 pc sample and its location about 3 mag above the WD sequence in the opticalto-NIR CMD (Fig. 9) and optical CMD (Fig. 10) requires an alternative explanation different from simple binarity.

An elusive class of WDs with low surface gravities of $5 \lesssim$ $\log g \lesssim 7$ and effective temperatures in the range of $8000 \mathrm{~K} \lesssim$ $T_{\text {eff }} \lesssim 22000 \mathrm{~K}$ are the so-called extremely low mass (ELM) WDs (Brown et al. 2016). With our estimated effective temperature of about $11800 \mathrm{~K}$ well within this interval, can the overluminosity of CA078 be explained with its possible ELM status? Our Balmer line equivalent width measurements (Figs. 2 and 3), in particular of the well-measured $\mathrm{H} \beta$ and $\mathrm{H} \gamma$ lines, already provided a hint on the possible low gravity $(\log g \lesssim 7)$ of CA078 (Sect. 4.2). All known DA3.6-DA4.5 shown (in Figs. 2 and 3 have much larger $\mathrm{H} \beta$ (and $\mathrm{H} \alpha$ ) line widths than $\mathrm{CA} 078$, whereas the $\mathrm{H} \gamma$ line widths are similar.

In addition to the Balmer line widths, we can also measure the gravity-dependent Balmer jump. We defined it as the flux ratio between the mean spectral energy densities in the wavelength intervals $4200-4240 \AA$ and $3700-3740 \AA$, which were covered both by the model and the observed spectra. Those flux ratios were also measured in spectra normalised to a continuum (instead of measuring in the original spectra) to avoid a colour term due to calibration uncertainties of the instrumental response function for our observed spectra. In Fig. 12 we compare the size of the Balmer jump in the observed and the model spectra. The graph indicates a low value of about $\log g=6$ of the surface gravity of CA078 with an upper limit of about $\log g=6.5$. This is consistent with an ELM status of CA078.

Gaia DR2 and SDSS data were already combined by Pelisoli et al. (2018a) in a study of so-called sdA stars, including (pre-)ELM WDs of low gravity and large radius. Using the same method, we estimate a radius of $0.0429_{-0.0122}^{+0.0213} r_{\odot}$ and a large uncertain distance of $913_{-403}^{+282} \mathrm{pc}$ for the known ELM SDSS J091709.55+463821.7 (Brown et al. 2016), which shows similar atmospheric parameters to CA078 $\left(\log g \approx 6\right.$ and $T_{\text {eff }} \approx$ $12000 \mathrm{~K})$. The estimated radius is about four times larger than 


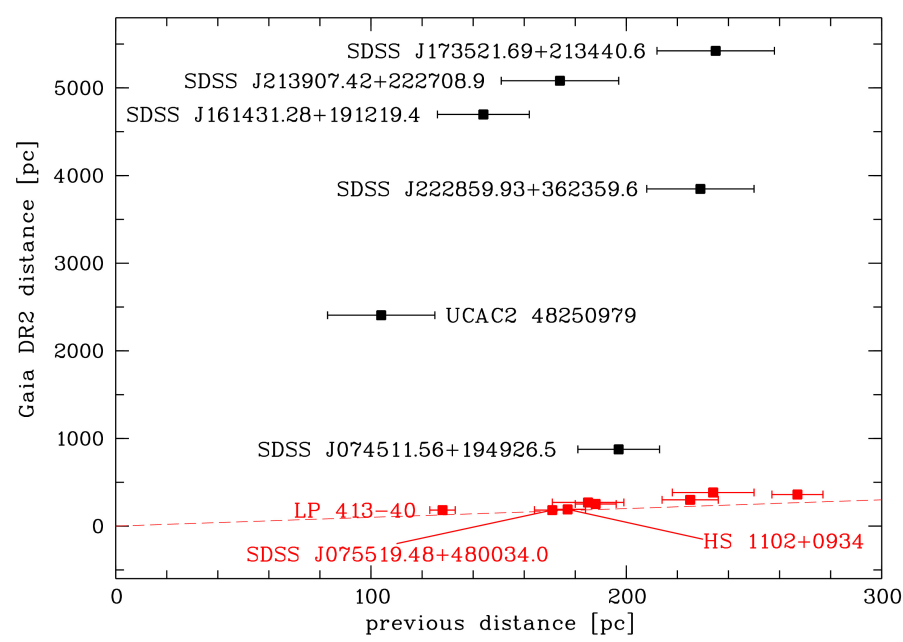

Fig. 13. Previous distances of the known nearest ELM WDs (see text) and their new distances derived from Gaia DR2 parallaxes. The red dashed line represents equality. Objects shifted by Gaia to much larger distances are labelled and shown in black. Other objects are shown in red, the three nearest of which are labelled.

the canonical WD radius corresponding to an increase in magnitude of about $3 \mathrm{mag}$, similar to what we see for CA078 in Figs. 9 and 10. While SDSS J091709.55+463821.7 has only an uncertain Gaia DR2 parallax and does not fall in the highquality Gaia DR2 100 pc sample, we can compute its absolute $G$ magnitude as about $9.1 \mathrm{mag}$, which is only slighly larger than that of $\mathrm{CA} 078(\approx 8.3 \mathrm{mag})$. Both objects have almost exactly the same zero colour indices $B P-R P 1$ and would appear very close to each other in the Gaia DR2 CMD of Fig. 10. Therefore, we conclude that CA078 is probably an ELM WD similar to SDSS J091709.55+463821.7 but at much smaller distance.

We have cross-identified the entire ELM survey sample of Brown et al. (2016) with Gaia DR2. Out of all 88 objects, 18 (with previous distance estimates between $0.42 \mathrm{kpc}$ and $7.77 \mathrm{kpc}$ ) have negative or no parallaxes measured in Gaia DR2. Out of the remaining 70 ELM WDs, there are only 14 previously considered as relatively nearby, with distances between $0.1 \mathrm{kpc}$ and $0.3 \mathrm{kpc}$ according to Brown et al. (2016). However, as can be seen in Fig. 13, six of them (shown in black) are shifted to much larger distances according to their Gaia DR2 parallaxes, including the previously assumed nearest ELM WD UCAC2 48250979. On the other hand, eight objects (shown in red) have only slightly larger Gaia DR2 distances compared to their previous distance estimates. The three nearest new ELM WDs are LP 413-40, SDSS J075519.48+480034.0, and HS 1102+0934 at distances of $182 \mathrm{pc}, 183 \mathrm{pc}$, and $191 \mathrm{pc}$. From these three objects, only LP 413-40 is included in the clean sample of ELM WD binaries (Brown et al. 2016) and lies with $B P-R P \approx+0.45 \mathrm{mag}$ and an absolute $\mathrm{G}$ magnitude of $\approx 10.34$ mag almost 3 mag above the normal WD sequence in the Gaia CMD (cf. Fig. 10), similar to CA078. We conclude that CA078, which is located about three times closer to the Sun and has a higher effective temperature than LP 413-40, represents a good new candidate for the nearest ELM WD.

\subsection{Outlook}

We have shown the Gaia DR2 colours $B P-R P$ to be a very effective tool for estimating effective temperatures of WDs. This can probably be further enhanced when a larger sample of known
WDs will be considered. The polynomial fitting with our small sample of known WDs already allowed us to reach uncertainties between $\pm 1100 \mathrm{~K}$ and $\pm 300 \mathrm{~K}$ for hot and cool WDs. We can for example estimate an effective temperature of $5100 \pm 300 \mathrm{~K}$ for the recently discovered new WD member of the $10 \mathrm{pc}$ sample, TYC 3980-1081-1 B (Scholz et al. 2018), based on its Gaia DR2 colour of $B P-R P \approx 1.06 \mathrm{mag}$.

We think that our new WDs, but also some of our rejected WDs, deserve further attention with follow-up observations, including higher-resolution spectroscopy, radial velocity monitoring, variability analysis, and imaging (to search for possible companions). In particular, the nature of the overluminous DA4. $2 \pm 0.5$ ELM WD candidate CA078 needs to be clarified. If confirmed, this would be the nearest ELM WD. The DB4.1 \pm 0.4 WD CA103 can be studied in more detail as one of the nearest representatives of its class. Among our rejected WDs, the G-type carbon dwarf CA171 is probably a promising target for highresolution spectroscopy, as it is as bright $(G \approx 13.35 \mathrm{mag})$ as the well-known and metal-poor $([\mathrm{Fe} / \mathrm{H}]=-4) \mathrm{dC}$ star $\mathrm{G} 77-61$. The latter is according to Margon \& Dimitriadis (2018) the only dC star with a detailed abundance analysis (Plez \& Cohen 2005) so far.

Acknowledgements. Most of our spectroscopic observations were made within a multi-season observing campaign carried out with the $2.2 \mathrm{~m}$ telescope of the Centro Astronómico Hispano-Aleman at Calar Alto, Spain. Part of these observations were carried out in service mode. We would like to thank the Calar Alto staff for their kind support and for their help with the observations. We thank Detlev Koester for providing his model spectra of DA WDs, Stephan Geier for helpful advice, and the anonymous referee for his kind report. We have extensively used SIMBAD and VizieR at the CDS/Strasbourg.

\section{References}

Berg, C., Wegner, G., Foltz, C. B., Chaffee, F. H., Jr., \& Hewett, P. C. 1992, ApJS, 78, 409

Brown, W. R., Gianninas, A., Kilic, M., Kenyon, S. J., \& Allende Prieto, C. 2016, ApJ, 818, 155

Drilling, J. S., Jeffery, C. S., Heber, U., Moehler, S., \& Napiwotzki, R. 2013, A\&A, 551, A31

Farihi, J., Wood, P. R., \& Stalder, B. 2005, ApJ, 627, L41

Favia, A., West, A. A., \& Theissen, C. A. 2015, ApJ, 813, 26

Finch, C., \& Zacharias, N. 2016a, ArXiv eprints [arXiv:1604. 06739]

Finch, C. T., \& Zacharias, N. 2016b, VizieR Online Data Catalog: I/333

Finch, C. T., \& Zacharias, N. 2018, VizieR Online Data Catalog: I/344

Finch, C. T., Zacharias, N., \& Jao, W.-C. 2018, AJ, 155, 176

Flynn, C., Holopainen, J., \& Holmberg, J. 2003, MNRAS, 339, 817

Gaia Collaboration (Babusiaux, C., et al.) 2018a, A\&A, 616, A10

Gaia Collaboration (Brown, A. G. A., et al.) 2018b, A\&A, 616, A1

Giammichele, N., Bergeron, P., \& Dufour, P. 2012, ApJS, 199, 29

Gianninas, A., Dufour, P., \& Bergeron, P. 2004, ApJ, 617, L57

Gianninas, A., Bergeron, P., \& Ruiz, M. T. 2011, ApJ, 743, 138

Gianninas, A., Curd, B., Thorstensen, J. R., et al. 2015, MNRAS, 449, 3966

Giclas, H. L., Burnham, R., Jr., \& Thomas, N. G. 1980, Lowell Observatory Bull., 8, 157

Green, P. 2013, ApJ, 765, 12

Greenstein, J. L. 1984, ApJ, 276, 602

Hansen, B. M. S. 1999, ApJ, 520, 680

Harris, H. C., Dahn, C. C., Subasavage, J. P., et al. 2018, AJ, 155, 252

Henden, A. A., Templeton, M., Terrell, D., et al. 2016, VizieR Online Data Catalog: II/336

Henry, T. J., Jao, W.-C., Winters, J. G., et al. 2018, AJ, 155, 265

Holberg, J. B., Barstow, M. A., \& Burleigh, M. R. 2003, ApJS, 147, 145

Holberg, J. B., Sion, E. M., Oswalt, T., et al. 2008, AJ, 135, 1225

Holberg, J. B., Oswalt, T. D., Sion, E. M., \& McCook, G. P. 2016, MNRAS, 462, 2295

Hollands, M. A., Koester, D., Alekseev, V., Herbert, E. L., \& Gänsicke, B. T. 2017, MNRAS, 467, 4970

Hollands, M. A., Tremblay, P.-E., Gänsicke, B. T., Gentile-Fusillo, N. P., \& Toonen, S. 2018, MNRAS, 480, 3942

Horne, K. 1986, PASP, 98, 609

Huber, D. \& Bryson, S. T. 2017, VizieR Online Data Catalog: IV/034 
Huber, D., Silva Aguirre, V., Matthews, J. M., et al. 2014, ApJS, 211, 2 Jahreiß, H., Meusinger, H., Scholz, R.-D., \& Stecklum, B. 2008, A\&A, 484, 575 Kawka, A., \& Vennes, S. 2012, MNRAS, 425, 1394

Kawka, A., Vennes, S., \& Thorstensen, J. R. 2004, AJ, 127, 1702

Kepler, S. O., Pelisoli, I., Koester, D., et al. 2015, MNRAS, 446, 4078

Kepler, S. O., Pelisoli, I., Koester, D., et al. 2016, MNRAS, 455, 3413

Kharchenko, N. V. 2001, Kinematika i Fizika Nebesnykh Tel, 17, 409

Kilic, M., Brown, W. R., \& McLeod, B. 2010, ApJ, 708, 411

Kilic, M., Munn, J. A., Harris, H. C., et al. 2017, ApJ, 837, 162

Kleinman, S. J., Kepler, S. O., Koester, D., et al. 2013, ApJS, 204, 5

Koester, D. 2010, Mem. Soc. Astron. It., 81, 921

Kupfer, T., Geier, S., Heber, U., et al. 2015, A\&A, 576, A44

Lépine, S., \& Shara, M. M. 2005, AJ, 129, 1483

Limoges, M.-M., Lépine, S., \& Bergeron, P. 2013, AJ, 145, 136

Limoges, M.-M., Bergeron, P., \& Lépine, S. 2015, ApJS, 219, 19

Lindegren, L., Lammers, U., Bastian, U., et al. 2016, A\&A, 595, A4

Lindegren, L., Hernández, J., Bombrun, A., et al. 2018, A\&A, 616, A2

Luo, A.-L., Zhao, Y.-H., Zhao, G., et al. 2015, Res. Astron. Astrophys., 15, 1095

Luyten, W. J. 1999, VizieR Online Data Catalog: III/070

Margon, B., \& Dimitriadis, G. 2018, Res. Notes Am. Astron. Soc., 2, 43

McCook, G. P., \& Sion, E. M. 1999, ApJS, 121, 1

Méndez, R. A. 2002, A\&A, 395, 779

Østensen, R. H., Oreiro, R., Solheim, J.-E., et al. 2010, A\&A, 513, A6

Osterbrock, D. E., \& Martel, A. 1992, PASP, 104, 76

Pelisoli, I., Bell, K. J., Kepler, S. O., \& Koester, D. 2018a, MNRAS, submitted [arXiv: 1805.04070 ]

Pelisoli, I., Kepler, S. O., \& Koester, D. 2018b, MNRAS, 475, 2480

Pickles, A., \& Depagne, É. 2010, PASP, 122, 1437

Plez, B., \& Cohen, J. G. 2005, A\&A, 434, 1117

Putney, A. 1997, ApJS, 112, 527

Randall, S. K., Fontaine, G., Geier, S., Van Grootel, V., \& Brassard, P. 2014, A\&A, 563, A79

Reed, M. D., Kilkenny, D., O’Toole, S., et al. 2012, MNRAS, 421, 181
Reid, I. N. 2005, ARA\&A, 43, 247

Reid, I. N., Sahu, K. C., \& Hawley, S. L. 2001, ApJ, 559, 942

Sayres, C., Subasavage, J. P., Bergeron, P., et al. 2012, AJ, 143, 103

Scholz, R.-D., Lehmann, I., Matute, I., \& Zinnecker, H. 2004, A\&A, 425, 519

Scholz, R.-D., Meusinger, H., \& Jahreiß, H. 2005, A\&A, 442, 211

Scholz, R.-D., Heber, U., Heuser, C., et al. 2015, A\&A, 574, A96

Scholz, R.-D., Meusinger, H., \& Jahreiß, H. 2018, A\&A, 613, A26

Sion, E. M., Greenstein, J. L., Landstreet, J. D., et al. 1983, ApJ, 269, 253

Sion, E. M., Holberg, J. B., Oswalt, T. D., et al. 2014, AJ, 147, 129

Skinner, J. N., Morgan, D. P., West, A. A., Lépine, S., \& Thorstensen, J. R. 2017, AJ, 154, 118

Skrutskie, M. F., Cutri, R. M., Stiening, R., et al. 2006, AJ, 131, 1163

Stellmacher, G., Wiehr, E., \& Grupe, D. 1992, A\&A, 265, 781

Subasavage, J. P., Henry, T. J., Bergeron, P., et al. 2007, AJ, 134, 252

Subasavage, J. P., Jao, W.-C., Henry, T. J., et al. 2009, AJ, 137, 4547

Subasavage, J. P., Jao, W.-C., Henry, T. J., et al. 2017, AJ, 154, 32

Toonen, S., Hollands, M., Gänsicke, B. T., \& Boekholt, T. 2017, A\&A, 602, A16 Tremblay, P.-E., \& Bergeron, P. 2009, ApJ, 696, 1755

Tremblay, P.-E., Kalirai, J. S., Soderblom, D. R., Cignoni, M., \& Cummings, J. 2014, ApJ, 791, 92

Tremblay, P.-E., Gentile-Fusillo, N., Raddi, R., et al. 2017, MNRAS, 465, 2849 Vennes, S., \& Kawka, A. 2012, ApJ, 745, L12

Vennes, S., Kawka, A., O’Toole, S. J., \& Thorstensen, J. R. 2012, ApJ, 756, L5

Wehrse, R., \& Liebert, J. 1980, A\&A, 86, 139

Wesemael, F., Greenstein, J. L., Liebert, J., et al. 1993, PASP, 105, 761

West, A. A., Morgan, D. P., Bochanski, J. J., et al. 2011, AJ, 141, 97

Xu, S., Ertel, S., Wahhaj, Z., et al. 2015, A\&A, 579, L8

York, D. G., Adelman, J., Anderson, J. E., Jr., et al. 2000, AJ, 120, 1579

Zacharias, N., Urban, S. E., Zacharias, M. I., et al. 2004, AJ, 127, 3043

Zacharias, N., Finch, C., Subasavage, J., et al. 2015, AJ, 150, 101

Zacharias, N., Finch, C., \& Frouard, J. 2017, AJ, 153, 166

Zhang, Y.-Y., Deng, L.-C., Liu, C., et al. 2013, AJ, 146, 34 